\title{
A Superconducting Instability in the Infinite-U Anderson Lattice in the Presence of Crystal Electric Fields
}

\author{
B. R. Trees* \\ Department of Physics \\ The Ohio State University \\ 174 W. 18th Ave. \\ Columbus, OH 43210 \\ January 24, 2017
}

\begin{abstract}
We report evidence of a superconducting instability (of $T_{1 g}$ symmetry) in the infinite$\mathrm{U}$ Anderson lattice in the presence of crystal fields of cubic symmetry. We assume a lattice of $4 f$ sites, each with a total angular momentum of $J=5 / 2$ that is split by crystal fields into a low-lying doublet of $\Gamma_{7}$ symmetry and an excited quartet of $\Gamma_{8}$ symmetry. Slave Bosons on the $4 f$ sites create and destroy $4 f^{0}$ configurations and Lagrange multipliers at each $4 f$ site enforce the occupancy constraint due to the infinite Coulomb repulsion. Quasiparticle interactions are due to exchange of $4 f$ density fluctuations, which are represented by fluctuations in the slave Bosons and Lagrange multipliers. We use the so-called analytic tetrahedron method to calculate the dressed (to order $1 / \mathrm{N}$ ) Boson Green functions. In weak couping, the exchange
\end{abstract}


of the dressed Bosons gives rise to a superconducting instability of $T_{1 g}, x y\left(x^{2}-y^{2}\right)$, symmetry. The $A_{1 g}$, "s-wave", channel has strongly repulsive interactions and hence no pairing instability. The $T_{2 g}$ channel exhibits weakly repulsive interactions. Average quasiparticle interactions in the $E_{g}, x^{2}-y^{2}, 3 z^{2}-r^{2}$, channel fluctuate strongly as a function of the number of tetrahedra used to calculate the Bosonic Green functions, lending only weak evidence for an instability of $E_{g}$ symmetry.

PACS No. 74.70.Tx 


\section{INTRODUCTION}

This paper is concerned with the effects of crystal electric fields on quasiparticle interactions in the Ce based heavy Fermion superconductor $\mathrm{CeCu}_{2} \mathrm{Si}_{2}\left(T_{c} \approx 0.6 \mathrm{~K}[1)\right.$. In general, the heavy Fermion materials are examples of systems exhibiting strong correlations among the constituent particles [2] [3], [4], [5], [6], [7]. Generally, the compounds are comprised of intermetallics and rare-earth or actinide atoms (such as uranium or cerium) with a strong on-site Coulomb repulsion. This large electrostatic energy arises from the extremely localized nature of the $4 \mathrm{f}$ or $5 \mathrm{f}$ wavefunctions in the solid and markedly influences the electron occupation at these "rare-earth" sites. When hybridization between a rare-earth electron and a conduction electron is allowed, the physics of this strong interaction is communicated to the solid at large, giving rise to a metal of strongly correlated, interacting electrons. In such a system one might expect to find a ground state manifesting collective properties of the coupled rare-earth and conduction electrons, e. g. superconductivity or magnetism. Indeed such ground states are seen. There are also heavy Fermion systems that apparently retain a metallic state down to zero temperature. To-date there are six known heavy Fermion superconductors, all containing either cerium or uranium.

Our work is based on the infinite-U Anderson lattice, the details of which we shall discuss later. For the experts, we mention here that we use slave Boson operators to create or destroy $4 f^{0}$ configurations on the Ce sites, thereby avoiding the cumbersome Hubbard operators in the hybridization piece of the Hamiltonian [8], [9], [10]. There is also a Lagrange multiplier to enforce unit occupancy of the $4 f$ multiplets at each Ce 
site. Our work is novel in that we also include, at the Ce sites, crystal electric fields of cubic symmetry, which has the effect of splitting the spin-orbit coupled $(J=5 / 2)$ multiplet into a doublet (of $\Gamma_{7}$ symmetry) and a quartet (of $\Gamma_{8}$ symmetry). We take the $\Gamma_{7}$ doublet to be the ground multiplet, with a crystal field splitting, $\Delta_{C E F}$, to the $\Gamma_{8}$ quartet that is much larger than the Kondo temperature of the low-lying doublet, $T_{o 7} \cdot\left(\Delta_{C E F} \gg T_{o 7}\right)$

Previous theoretical work has focused on understanding the heavy Fermion compounds mainly through the $S U(N)$ version of the periodic Anderson model [11], [12], [13], [14], [15]. In the $S U(N)$ model, the $4 f$ multiplet is $N$-fold degenerate, and the (planewave) conduction bands are assumed $N$-fold degenerate as well. The matrix element, $V(\vec{k})$, for hybridization between a conduction electron and a $4 f$ electron, is taken to be isotropic in momentum space.

Within the $S U(N)$ model, Lavagna, Millis, and Lee[15], Auerbach and Levin [12], and Houghton, Read, and Won 16 have studied quasiparticle interactions due to the exchange of $4 f$ density fluctuations. The lowest order diagrams contributing to the interactions are of order $1 / N$, where $N$ is the $4 f$ multiplet degeneracy. In Ce, in the absence of crystal field splitting, the low-lying $J=5 / 2$ multiplet is six-fold degenerate $(N=6)$. So it seems reasonable to truncate the diagrams at order $1 / N$. Lavagna, Millis, and Lee found such a spinless density exchange yielded a $d$-wave superconducting instability in the spin-singlet pairing channel.

F. C. Zhang and T. K. Lee 17 have performed a more realistic calculation (as far as heavy Fermion compounds are concerned) by including spin-orbit coupling at the Ce sites and by returning to the two-fold degenerate conduction states. They included an 
anisotropic hybridization matrix element, of the Coqblin-Schrieffer form [18, between conduction and $4 f$ electrons. These spin-orbit coupled ions are assumed to sit in an overall spherically symmetric "host" (as in a jellium model). Unlike in the $S U(N)$ model, in the even-parity pairing channel, Zhang and Lee found no superconducting instabilities of $s$-wave, $d$-wave, or $g$-wave symmetry. It is worth noting here that the mean field quasiparticle energy bands Zhang and Lee found are the same as those calculated by Zou and Anderson[19], who used the KKR scheme and included spinorbit coupling on the $4 f$ sites.

We have looked at density-fluctuation induced quasiparticle interactions in the presence of crystal electric field splitting of the Ce $J=5 / 2$ multiplets in the lattice. We find the anisotropy due to cubic symmetry qualitatively and quantitatively alters the interactions in comparison to the results of Zhang and Lee. In fact, we find evidence for a superconducting instability of $T_{1 g}\left(x y\left(x^{2}-y^{2}\right)\right)$ symmetry in the even-parity pairing channel. We also find weaker evidence for an $E_{g}\left(x^{2}-y^{2}\right)$ pairing instability. We find no instability in the $A_{1 g}$ (the "s-wave" of cubic symmetry) channel, which is not surprising, given the strong Hubbard U repulsion built into the Anderson model.

The details of our calculation are presented here as follows. In Sec. II, we briefly discuss experimental evidence for the existence of crystal electric fields of cubic symmetry in $\mathrm{CeCu}_{2} \mathrm{Si}_{2}$. In Sec. III, we introduce the Hamiltonian for the infinite$\mathrm{U}$ Anderson lattice in the presence of crystal fields. The Hamiltonian formalism shall be retained throughout this paper, as opposed to functional integral techniques [9], [12], [14], [15], [20]. In Sec. IV, we report the mean field properties of our Hamil- 
tonian, including the quasiparticle energies and states. In Sec. VI, we discuss our calculation of the dressed slave Boson Green functions, which include the effects of particle-hole excitations in the hybridization coupled conduction and $4 f$ electron system. We also explain (Secs. VII and VIII) our use of the so-called analytic tetrahedron method for performing the complicated three dimensional Brillouin zone integrals that arise in the evaluation of the particle-hole diagrams. Finally, in Secs. X and XI, we present our results for the superconducting instabilities in the presence of cubic symmetry, which are based on the Fermi surface average of the quasiparticle-quasiparticle scattering amplitude.

\section{CRYSTAL ELECTRIC FIELDS}

The first experimental evidence of crystal electric fields in $\mathrm{CeCu}_{2} \mathrm{Si}_{2}$ came from the inelastic neutron scattering data of Horn et al. [21]. The data show a clear peak at an energy of $31.5 \mathrm{meV}(\approx 360 \mathrm{~K})$ and a weaker peak at approximately $12 \mathrm{meV}$. Since there was also some structure at $12 \mathrm{meV}$ in the reference material $\mathrm{LaCu}_{2} \mathrm{Si}_{2}$ (which has no $4 f$ electrons), it seems likely that the lower energy peak is due to phonons. The data could be fit, however, by assuming a crystal field structure in which the 6-fold degenerate $J=5 / 2$ multiplets are split into three doublets, with excitation energies given by the measured energies of the inelastic peaks. Such a three doublet structure is exactly what one would expect for a tetragonal crystal, like $\mathrm{CeCu}_{2} \mathrm{Si}_{2}$.

Later, measurements of the specific heat at high temperatures [22], however, did not agree with this interpretation of the multiplet structure. Bredl et al. plotted the $4 f$ contribution to the specific heat of $\mathrm{CeCu}_{2.2} \mathrm{Si}_{2}$ by subtracting off the corresponding 
data for $\mathrm{LaCu}_{2.2} \mathrm{Si}_{2}$. They found they could not fit the results with two Schottky peaks, as would be expected for three crystal field doublets. Instead, the excited magnetic states behaved as if there were a four-fold degenerate multiplet about 360 K above a ground state doublet. This idea is also nicely corroborated by Bredl and co-workers' plot of the entropy as a function of temperature. The rather quick rise of the entropy from a value of $R \ln 2$ to $R \ln 6$ at about $300 \mathrm{~K}$ would suggest a quartet structure rather than two doublets separated in energy by about 100K. Such a structure would be appropriate in the presence of cubic symmetry.

Further evidence for effective cubic symmetry in $\mathrm{CeCu}_{2} \mathrm{Si}_{2}$ comes from the dc susceptibility measurements by Steglich and co-workers on single crystal $\mathrm{CeCu}_{2} \mathrm{Si}_{2}$ samples 23. They find a very weak anisotropy in the temperature dependence of $\chi_{d c}$ for a magnetic field applied parallel or perpendicular to the c-axis of the unit cell. Furthermore, the isotropy of the slope of the upper critical magnetic field at $T_{c}$, $H_{c 2}^{\prime}\left(T_{c}\right)$ [24], suggests that the effective mass of the quasiparticles is isotropic. Since the effective mass is technically a second-rank tensor, it can be isotropic only in the presence of cubic symmetry. In light of all this information, it is reasonable to assume that the small peak in the neutron data at $12 \mathrm{meV}$ is not due to crystal field excitations and that there is effectively cubic symmetry at the cerium sites.

In the presence of cubic symmetry, the $\mathrm{J}=5 / 2$ multiplet is split into a doublet of $\Gamma_{7}$ symmetry and a $\Gamma_{8}$ quartet [25]. The crystal field-split states $|\Gamma \alpha\rangle$, where $\alpha$ labels the degenerate states for a given multiplet, are a linear combination of the eigenstates of the z-component of the total angular momentum, $|m\rangle$, where $-5 / 2 \leq m \leq 5 / 2$ :

$$
\left|\Gamma_{7},+1\right\rangle=-\sqrt{\frac{1}{6}}|-5 / 2\rangle+\sqrt{\frac{5}{6}}|3 / 2\rangle
$$




$$
\begin{gathered}
\left|\Gamma_{7},-1\right\rangle=-\sqrt{\frac{1}{6}}|5 / 2\rangle+\sqrt{\frac{5}{6}}|-3 / 2\rangle \\
\left|\Gamma_{8},+2\right\rangle=\sqrt{\frac{5}{6}}|5 / 2\rangle+\sqrt{\frac{1}{6}}|-3 / 2\rangle \\
\left|\Gamma_{8}, 1\right\rangle=|1 / 2\rangle \\
\left|\Gamma_{8},-1\right\rangle=|-1 / 2\rangle \\
\left|\Gamma_{8},-2\right\rangle=\sqrt{\frac{5}{6}}|-5 / 2\rangle+\sqrt{\frac{1}{6}}|3 / 2\rangle .
\end{gathered}
$$

From the neutron scattering and specific heat data we know that the ground multiplet is the doublet. Thus, without addressing the true microscopic source of the crystal fields, we deduce a multiplet structure as shown in figure 1, where $\Delta_{C E F}$ labels the size of the crystal field splitting. In general, equations 1 - 6 can be expressed as

$$
|\Gamma, \alpha\rangle=\sum_{m} c_{\Gamma \alpha m}|m\rangle
$$

where the coefficients $c_{\Gamma \alpha m}$ can be read directly from the equations.

We can use equation 0 to construct the matrix element, $\mathrm{V}_{\Gamma \alpha \sigma}(\vec{k})$, for hybridization between a crystal field state with quantum numbers $\Gamma, \alpha$ and a plane wave conduction state with crystal momentum $\vec{k}$ and spin $\sigma$. In the case of a single ion in the full $\mathrm{J}=5 / 2$ manifold, one may use the form derived by Coqblin and Schrieffer [18],

$$
V_{m \sigma}(\vec{k})=-\sqrt{\frac{4 \pi}{3}}(-i)^{3} \sigma V_{o k} \sqrt{\frac{7-2 m \sigma}{14}} Y_{3, m-\frac{\sigma}{2}}^{*}(\hat{k}),
$$

$(-5 / 2 \leq m \leq 5 / 2), \sigma= \pm 1$ is the (pseudo)spin index, and the angular dependence is in the spherical harmonic. $V_{o k}$ denotes the dependence of the hybridization strength on the magnitude of the momentum, which will be important only near the zone center (i.e. near $|k|=0)$. In fact, we can write $V_{o k}=V_{o} g(k)$, where $g(k)$ is a function 
of $|\vec{k}|$ that goes to zero at the zone center like $k^{3} . V_{o}$ is the bare hybridization strength. Because the crystal field split $4 f$ states are just linear combinations of the $J=5 / 2$ states, the hybridization matrix elements in cubic symmetry are

$$
V_{\Gamma \alpha \sigma}(\vec{k})=\sum_{m=-5 / 2}^{5 / 2} c_{\Gamma \alpha m} V_{m \sigma}(\vec{k}) .
$$

Figure 2 represents the hybridization process pictorially.

\section{HAMILTONIAN}

We start with a few words on the important energy scales of $\mathrm{CeCu}_{2} \mathrm{Si}_{2}$, based on the discussion of Kang et al. 26] on the electron spectroscopic data available as of 1990. They analyzed their own data of the Ce $3 d$ x-ray photoelectron spectrum (XPS) and $4 f$ bremsstrahlung isochromat spectrum (BIS); they also analyzed Ce $4 f$ resonant photoelectron data (RESPES) of Parks et al. 27. Kang and co-workers calculated the appropriate one-electron spectra from the impurity Anderson model, which showed reasonably good agreement with the data. On the basis of such a calculation, the authors claim that Coulomb energy for double occupation of a Ce $4 f$ site is $U \approx 7 \mathrm{eV}$.

The large Coulomb energy for $\mathrm{CeCu}_{2} \mathrm{Si}_{2}$ prompts us to take the limit in which $U$ goes to infinity, thereby forbidding hybridization processes that give rise to $4 f^{1} \rightarrow$ $4 f^{2}$ valence fluctuations. This is a technical simplification for us, but even though $U$ is indeed very large by solid-state physics standards, it may be that the physics of finite $U$ is crucial to the understanding of heavy Fermions. Recently Dan Cox 28] has proposed the Quadrupolar Kondo Model (or Two-Channel Kondo Model) as an 
explanation of the superconductivity and of the possible non-Fermi liquid behavior in uranium based heavy Fermions. The physics needed to get non-Fermi liquid behavior derives from the existence of enough independent channels of conduction electrons to overcompensate the effective $4 f$ "spin". A finite- $U$ version of the Anderson model, as applied to a Ce impurity, can exhibit a two-channel Kondo effect. The crystal field split multiplet structure here is the key. Taking the $\Gamma_{7}$ doublet as the low-lying multiplet for the $4 f^{1}$ configuration, and a $\Gamma_{3}$ (non-magnetic) doublet as the low lying multiplet for the $4 f^{2}$ configuration (as is appropriate for cubic symmetry), a twochannel Kondo effect is possible. Conduction states of $\Gamma_{8}$ symmetry can mix the $4 f^{1}$ and $4 f^{2}$ configurations 29, and conduction states of $\Gamma_{7}$ symmetry can mix the $4 f^{1}$ and $4 f^{0}$ configurations. If the hybridization of $\Gamma_{8}$ conduction states is stronger than hybridization of $\Gamma_{7}$ conduction states, the physics of the two-channel Kondo effect will determine the low temperature properties of the model. It may be that the finite-U Anderson model is a better starting point for heavy fermion systems. Nevertheless, the infinite $U$ limit is a reasonable simplification (at least for $\mathrm{CeCu}_{2} \mathrm{Si}_{2}$ ) of an already difficult problem and warrants study in its own right.

Our Hamiltonian can be written as a combination of terms: $H=H_{c}+H_{f}+$ $H_{m i x}+H_{\text {constraint }}$. The kinetic energy of the conduction electrons is given by,

$$
H_{c}=\sum_{\vec{k} \sigma} \xi_{\vec{k}} c_{\vec{k} \sigma}^{\dagger} c_{\vec{k} \sigma}
$$

where

$$
\xi_{\vec{k}}=\frac{\hbar^{2} k^{2}}{2 m}-\mu_{o}
$$

is the plane-wave dispersion. The zero of energy for this calculation will be taken 
with respect to $\mu_{o}$, the chemical potential of the conduction electrons in the absence of hybridization.

The $4 f$ electron site energy is

$$
H_{f}=\sum_{\vec{R} \Gamma \alpha} E_{\Gamma} f_{\vec{R} \Gamma \alpha}^{\dagger} f_{\vec{R} \Gamma \alpha}
$$

where $\vec{R}$ is the site index in real-space, and $\Gamma \alpha$ are the crystal field quantum numbers. The operator $f_{\vec{R} \Gamma \alpha}$ destroys a $4 f^{1}$ configuration at lattice site $\vec{R}$, in which the crystal field state $\Gamma \alpha$ is initially occupied. From the photoemission data [26], we take the energy of the $\Gamma_{7}$ doublet to be $-2.0 \mathrm{eV}$, i.e. $E_{7}=-2.0 \mathrm{eV}$. From the inelastic neutron scattering data[21] and the high temperature specific heat 22], we take the $\Gamma_{8}$ level to lie $360 \mathrm{meV}$ above the $\Gamma_{7}$ level,

$$
E_{8}=E_{7}+\Delta_{C E F}=-1.964 \mathrm{eV} .
$$

The hybridization, or mixing, term is

$$
H_{m i x}=\frac{1}{\sqrt{N_{s}}} \sum_{\vec{k} \sigma \vec{R} \Gamma \alpha}\left[V_{\Gamma \alpha \sigma}(\vec{k}) c_{\vec{k} \sigma}^{\dagger} f_{\vec{R} \Gamma \alpha} b_{\vec{R}}^{\dagger} e^{i \vec{k} \cdot \vec{R}}+H . c .\right] .
$$

The operator $b_{\vec{R}}^{\dagger}$ is a slave Boson creation operator, which creates a $4 f^{0}$ configuration, or hole, at lattice site $\vec{R}$. $N_{s}$ is the number of lattice sites. This combination of conduction, $4 f$, and slave Boson operators was first applied to the Anderson model by Barnes 8], and was later reintroduced by Coleman 10. $H_{m i x}$ contains only Bosonic or Fermionic operators, and so Wick's theorem is applicable. Use of the more cumbersome Hubbard projection operators,

$$
X_{0 \Gamma \alpha}=|0\rangle\langle\Gamma \alpha|
$$


would make Feynman diagrammatic procedures invalid, since the Hubbard operators do not obey standard commutation relations. The cubic symmetry is reflected in the structure of the anisotropic function $\mathrm{V}_{\Gamma \alpha \sigma}(\vec{k})$, which has a significantly different $\vec{k}$ dependence than the Coqblin-Schrieffer form $\mathrm{V}_{m \sigma}(\vec{k})$. This new anisotropy will affect quasiparticle interactions differently than in the case of spherical symmetry.

Finally, there is the constraint term, which is introduced with a Lagrange multiplier, $i \lambda_{\vec{R}}$, insuring that the total occupancy (Fermions plus Bosons) at the Ce sites is unity,

$$
H_{\text {constraint }}=\sum_{\vec{R}} i \lambda_{\vec{R}}\left(f_{\vec{R} \Gamma \alpha}^{\dagger} f_{\vec{R} \Gamma \alpha}+b_{\vec{R}}^{\dagger} b_{\vec{R}}-Q\right) .
$$

Note that $Q=1$ is the physically meaningful value in this case.

There are a few technical points we need to mention here. As discussed by Read and Newns [9] in the functional integral approach to the infinite-U Anderson impurity, it is useful to write the slave Boson operator as the product of a modulus and a phase factor, which in the lattice problem picks up a site index, $\vec{R}$,

$$
b_{\vec{R}}=s_{\vec{R}} e^{i \theta_{\vec{R}}} .
$$

A gauge transformation for the $f$ operators,

$$
f_{\vec{R} \Gamma \alpha} \rightarrow f_{\vec{R} \Gamma \alpha} e^{-i \theta_{\vec{R}}}
$$

then absorbs all the phase factors. In the functional integral approach, furthermore, the Lagrangian corresponding to our Hamiltonian (equations 10, 12, 14, and 16) contains an (imaginary) time derivative of the slave Bosons,

$$
b_{\vec{R}}^{*}(\tau) \frac{\partial b_{\vec{R}}(\tau)}{\partial \tau},
$$


which, from equation 17, introduces a factor of the phase velocity, $\dot{\theta}_{\vec{R}}$, into the Lagrangian. Traditionally, this phase velocity is absorbed into the Lagrange multiplier, elevating it to the status of a dynamical field, $i \lambda_{\vec{R}}(\tau)$. In this paper, since we have used the Hamiltonian formalism, we remark that, in the limit of static slave Bosons, the Lagrange multiplier plays the same role as it does in the functional integral formalism[11]. We have a $2 \times 2$ matrix Green function for the slave Boson and Lagrange multiplier, which will have elements composed of averages over the following combinations of fields: $s s, s \lambda$, and $\lambda \lambda$.

Our calculation is based on previous $1 / N$ calculations of the type applied to the $S U(N)$ model, where $N$ is the degeneracy of the $4 f$ multiplet. In the $S U(N)$ case, in order to have a well defined Kondo temperature in the limit of large $N$, it was necessary to assume the bare hybridization strength, $V_{o}$, scaled like $1 / \sqrt{N}[11]$. We do the same here by defining a rescaled hybridization matrix element,

$$
\tilde{V}_{\Gamma \alpha \sigma}(\vec{k}) \equiv \sqrt{N_{\Gamma}} V_{\Gamma \alpha \sigma}(\vec{k}),
$$

where we assume that $\tilde{V}_{\Gamma \alpha \sigma}(\vec{k})$ is of order 1 . Note that $N_{\Gamma}$ is the degeneracy of the $\Gamma$ crystal field multiplet. Also, because the hybridization matrix element and the Boson operator, $s_{\vec{R}}$, appear together in $H_{m i x}$, we define a scaled Boson operator,

$$
\tilde{s}_{\vec{R} \Gamma} \equiv \frac{s_{\vec{R}}}{\sqrt{N_{\Gamma}}},
$$

where we assume that $\tilde{s}_{\vec{R} \Gamma}$ is also of order 1 .

\section{MEAN FIELD APPROXIMATION}


In this section, we discuss the properties of our Hamiltonian at mean field level, where we assume both the Bose operator and the Lagrange multiplier are uniform in space. In this limit, the Hamiltonian in k-space takes the following form:

$$
\begin{gathered}
H_{M F}=\sum_{\vec{k} \sigma} \xi_{\vec{k}} c_{\vec{k} \sigma}^{\dagger} c_{\vec{k} \sigma}+\sum_{\vec{k} \Gamma \alpha} \epsilon_{\Gamma} f_{\vec{k} \Gamma \alpha}^{\dagger} f_{\vec{k} \Gamma \alpha} \\
+\sum_{\vec{k} \sigma \Gamma \alpha}\left[\tilde{s}_{o \Gamma} \tilde{V}_{\Gamma \alpha \sigma}(\vec{k}) c_{\vec{k} \sigma}^{\dagger} f_{\vec{k} \Gamma \alpha}+H . c .\right] \\
\quad+\frac{N_{s}}{2} \sum_{\Gamma} N_{\Gamma} i \lambda_{o}\left(\tilde{s}_{o \Gamma}^{2}-q_{o \Gamma}\right) .
\end{gathered}
$$

where $\epsilon_{\Gamma} \equiv E_{\Gamma}+i \lambda_{o}$ is the shifted energy of the $\Gamma$ multiplet. We assume that $q_{o \Gamma}$ is of order 1 , but technically, when it comes down to getting numerical results, we know that $q_{o \Gamma}=1 / N_{\Gamma}$. Note that $\tilde{s}_{o \Gamma}$ and $\lambda_{o}$ are the mean field values of the Bose operator and Lagrange multiplier, respectively $\left(\tilde{s}_{O \Gamma}=s_{o} / \sqrt{N_{\Gamma}}\right)$.

It is straightforward to diagonalize $H_{M F}$ and obtain the quasiparticle states and energies. Because the $\Gamma_{8}$ states are four-fold degenerate, there is one non-hybridizing quasiparticle band of $\Gamma_{8}$ symmetry. The secular equation which gives the quasiparticle energies $E_{n \vec{k}}(n=1,2,3$ is the band index $)$ is

$$
\left(\epsilon_{8}-E_{n \vec{k}}\right)\left[\left(\xi_{\vec{k}}-E_{n \vec{k}}\right)-\sum_{\alpha_{7}} \frac{\tilde{s}_{o 7}^{2}\left|\tilde{V}_{7 \alpha \sigma}\right|^{2}}{\left(\epsilon_{7}-E_{n \vec{k}}\right)}-\sum_{\alpha_{8}} \frac{\tilde{s}_{o 8}^{2}\left|\tilde{V}_{8 \alpha \sigma}\right|^{2}}{\left(\epsilon_{8}-E_{n \vec{k}}\right)}\right]=0,
$$

where the sum $\sum_{\alpha_{7}(8)}$ means only the states in the $\Gamma_{7(8)}$ doublet are summed. Although technically it is possible to find analytic solutions of equation 21, the analytic expressions are too cumbersome to be useful; thus we calculated the roots numerically. The three quasiparticles bands are plotted in figure 3 along two different directions in the cubic Brillouin zone. Figure 3 shows that along the axes of the zone, for example along the $\Gamma X$ direction, the states of $\Gamma_{7}$ symmetry can not hybridize with the 
conduction states. This means that the matrix elements $V_{7 \alpha \sigma}(\vec{k})$ vanish along these special directions and that there is no gap between the first and second quasiparticle bands (see figure 3(b)). Such behavior has been discussed by Martin [30].

The quasiparticle states can, quite generally, be written as a combination of plane wave and crystal field states,

$$
\left|Q_{\vec{k} n \sigma}\right\rangle=A_{n}(\vec{k})\left[|\vec{k} \sigma\rangle-\sum_{\Gamma \alpha} \frac{\tilde{s}_{o \Gamma} \tilde{V}_{\Gamma \alpha \sigma}^{*}(\vec{k})}{\epsilon_{\Gamma}-E_{n \vec{k}}}|\Gamma \alpha\rangle\right],
$$

where $|\vec{k} \sigma\rangle$ is a plane wave state. The anisotropic normalization function is

$$
A_{n}^{2}(\vec{k})=\left[1+\frac{1}{2} \sum_{\Gamma \alpha \sigma} \frac{\tilde{s}_{o \Gamma}^{2}\left|\tilde{V}_{\Gamma \alpha \sigma}(\vec{k})\right|^{2}}{\left(\epsilon_{\Gamma}-E_{n \vec{k}}\right)^{2}}\right]^{-1} .
$$

For given crystal field quantum numbers, $\Gamma \alpha$, it is possible to sum over the pseudospin variable $\sigma$ in equation 23 . For convenience, we define a function

$$
\mu_{\Gamma \alpha \Gamma^{\prime} \alpha^{\prime}}(\vec{k}) \equiv \sum_{\sigma} \tilde{V}_{\Gamma \alpha \sigma}^{*}(\vec{k}) \tilde{V}_{\sigma \Gamma^{\prime} \alpha^{\prime}}(\vec{k})
$$

Then the sum over the pseudospin variable in equation 23 is just the diagonal element $\mu_{\Gamma \alpha \Gamma \alpha}(\vec{k})$. All possible (non-zero) forms for the function $\mu_{\Gamma \alpha \Gamma^{\prime} \alpha^{\prime}}(\vec{k})$ are shown in Table 1 , where the dependence on the magnitude of $\vec{k}$ has been divided out. Using the results of this table, it is possible to arrive at the following expression for the normalization function, which is valid at any point in the Brillouin zone:

$$
A_{1}^{2}=\frac{\left(\frac{T_{o 7}}{s_{o} V_{o}}\right)^{2}}{\frac{1}{3}+\frac{2}{3}\left(\frac{T_{o 7}}{T_{o 8}}\right)^{2}-\frac{2 \sqrt{\pi}}{9}\left[1-\left(\frac{T_{o 7}}{T_{o 8}}\right)^{2}\right]\left[Y_{40}(\hat{k})+\sqrt{\frac{5}{14}}\left(Y_{44}(\hat{k})+Y_{4-4}(\hat{k})()\right]\right.} .
$$

Figure 4 shows a plot of equation 25 along the equator of a spherical Fermi surface, with $\phi$ denoting the azimuthal angle measured with respect to a coordinate axis. The 
extremely sharp variations near the axes represent the vanishing of the $\Gamma_{7}$ hybridization matrix elements. We call the six points where the Brillouin zone axes intersect the Fermi surface "hot-spots"; they must be handled with care when averaging the quasiparticle scattering amplitude over the Fermi surface.

We would like to remark that, in this model, the existence of "hot-spots" is a manifestation of the lowering of the symmetry below spherical. In spherical symmetry, there is a sum rule for the matrix elements that renders the normalization function, $A^{2}$, isotropic in k-space[15], 117. In our case, the matrix elements can not be simplified to an isotropic function. This means that "hot-spots" can occur anywhere in the Brillouin zone where some subset of the hybridization matrix elements, $\tilde{V}_{\Gamma \alpha \sigma}(\vec{k})$, vanishes. This is a rather general statement that relies only on the symmetry being lower than spherical and should not be unique to cubic lattices.

We can relate the fermionic creation and destruction operators in the original basis (the $c$ and $f$ operators) to creation and destruction operators in the quasiparticle basis, $Q_{\vec{k} n \sigma}^{\dagger}, Q_{\vec{k} n \sigma}$. We find that

$$
\begin{gathered}
c_{\vec{k} \sigma}=\sum_{n} A_{n}(\vec{k}) Q_{\vec{k} n \sigma}, \\
f_{\vec{k} \Gamma \alpha}=-\sum_{n \sigma} \frac{A_{n}(\vec{k}) \tilde{s}_{o \Gamma} \tilde{V}_{\Gamma \alpha \sigma}^{*}(\vec{k})}{\epsilon_{\Gamma}-E_{n \vec{k}}} Q_{\vec{k} n \sigma} .
\end{gathered}
$$

These expressions are useful in constructing the two-quasiparticle scattering amplitude.

In the last part of this section, we discuss the self-consistency equations that arise when one demands that the free energy in the mean field approximation be an extremum with respect to the Bose fields $s_{o}$ and $i \lambda_{o}$. Since we have diagonalized the 
mean field Hamiltonian, equation 20, we can write it in terms of the quasiparticle energies and operators:

$$
H_{M F}=\sum_{\vec{k} n \sigma} E_{n \vec{k}} Q_{\vec{k} n \sigma}^{\dagger} Q_{\vec{k} n \sigma}+\frac{N_{s}}{2} \sum_{\Gamma} N_{\Gamma} i \lambda_{o}\left(\tilde{s}_{o \Gamma}^{2}-q_{o \Gamma}\right) .
$$

The corresponding mean field free energy has the form

$$
F_{M F}=\frac{N_{s}}{2} \sum_{\Gamma} N_{\Gamma} i \lambda_{o}\left(\tilde{s}_{o \Gamma}^{2}-q_{o \Gamma}\right)-\frac{1}{\beta} \sum_{\vec{k} n \sigma} \ln \left(1+e^{-\beta E_{n \vec{k}}}\right),
$$

where $\beta$ is the inverse temperature. Requiring that $\partial F_{M F} / \partial i \lambda_{o}=0$ and $\partial F_{M F} / \partial s_{o}=0$ yields the equations

$$
\frac{1}{2} \sum_{\Gamma} N_{\Gamma}\left(\tilde{s}_{o \Gamma}^{2}-q_{o \Gamma}\right)+\frac{1}{N_{s}} \sum_{\vec{k} \sigma n} f\left(E_{n \vec{k}}\right) \frac{\partial E_{n \vec{k}}}{\partial i \lambda_{o}}=0
$$

and

$$
2 i \lambda_{o} s_{o}+\frac{1}{N_{s}} \sum_{\vec{k} \sigma n} f\left(E_{n \vec{k}}\right) \frac{\partial E_{n \vec{k}}}{\partial s_{o}}=0 .
$$

In equations 30 and $31 f\left(E_{n \vec{k}}\right)$ is the Fermi function evaluated at the quasiparticle energy $E_{n \vec{k}}$. We also need an equation to fix the chemical potential of the quasiparticles, $\mu$, which depends on the total number of electrons (conduction electrons, $n_{c}$, and f electrons, $n_{f}$ ) per unit cell

$$
n_{\text {total }}=n_{c}+n_{f}=\frac{1}{N_{s}} \sum_{\vec{k} \sigma n} f\left(E_{n \vec{k}}\right)
$$

These three coupled integral equations, when solved self-consistently, give the shifted $4 \mathrm{f}$ multiplet energies, $\epsilon_{\Gamma}$, the value of the Bose field, $s_{o}$, and the quasiparticle chemical potential, $\mu$. The input parameters are $n_{\text {total }}$, the quasiparticle filling factor, the bare hybridization strength, $V_{o}$, and the conduction electron filling factor, $n_{c}$. The 
zero of energy is always measured relative the the chemical potential of the conduction electrons, $\mu_{o}$. For $n_{\text {total }}=2$, the lowest quasiparticle band is completely filled, and the system is a Kondo insulator. We have consistently used $n_{\text {total }}=1.5$, which insures that we have a metal.

To get numerical self-consistent solutions, we found it necessary to write these equations in terms of energy integrals with the appropriate density of states,

$$
\frac{1}{N_{s}} \sum_{\vec{k} \sigma} \rightarrow 2 \int d \xi N(\xi) \int \frac{d \Omega}{4 \pi},
$$

where $N(\xi)$ is the density of states per spin for the unhybridized conduction electrons and $\mathrm{d} \Omega$ is an element of solid angle. Note that in the $S U(N)$ model described previously, the spin degeneracy would contribute a prefactor of $N$ (instead of 2) in equation 33. For free electrons in three dimensions the density of states is proportional to the square root of $\xi$.

To proceed, we make one approximation. We assume that surfaces of constant energy for the quasiparticle states are spherically symmetric. Near the zone center, this is exactly correct, and there is no approximation at all. Near the zone boundary, the equal-energy surfaces become distorted from spheres due to the constraints of $\Gamma_{7}$ symmetry. It is important to note, however, that in equations 30, 31, and 32, the strongest angular dependence comes from the anisotropic matrix elements, which we treat exactly. That is, we believe the angular dependence of the quasiparticle bands is not as important as that of the hybridization matrix elements. For example, near an axis of the Brillouin zone, the $\Gamma_{7}$ matrix elements are going to zero. So, even if the quasiparticle energies surfaces are distorted from spheres near the axes, the sensitivity of the self-consistency equations to this distortion would be lessened by the presence of 
the small $V_{7 \alpha \sigma}$ terms in the numerator. Thus, it should be a reasonable approximation to treat the mixing matrix elements as having all the angular dependence.

Using the secular equation for the quasiparticle band energies, equation 21, we can calculate all the necessary derivatives of the quasiparticle energies found in equations 30 and 31. After averaging the anisotropic matrix elements over the Fermi surface, we are left with the following three equations to be solved self-consistently:

$$
\begin{gathered}
\frac{1}{2} \sum_{\Gamma} N_{\Gamma}\left(\tilde{s}_{o \Gamma}^{2}-q_{o \Gamma}\right)+2 \frac{s_{o}^{2} V_{k o}^{2}}{3} \int_{-D}^{\mu} d E N(\xi(E))\left[\frac{1}{\left(\epsilon_{7}-E\right)^{2}}+\frac{5}{\left(\epsilon_{8}-E\right)^{2}}\right]=0 \\
2 i \lambda_{o}-2 \frac{V_{k o}^{2}}{3} \int_{-D}^{\mu} d E N(\xi(E))\left[\frac{1}{\epsilon_{7}-E}+\frac{5}{\epsilon_{8}-E}\right]=0, \text { and } \\
n_{\text {total }}-2 \int_{-D}^{\mu} d E N(\xi(E))\left[1+\frac{1}{3} \frac{s_{o}^{2} V_{o k}^{2}}{\left(\epsilon_{7}-E\right)^{2}}+\frac{5}{3} \frac{s_{o}^{2} V_{o k}^{2}}{\left(\epsilon_{8}-E\right)^{2}}\right]
\end{gathered}
$$

where $-D$ is the energy at the bottom of the lowest quasiparticle band.

We have taken the limit of zero temperature to arrive at equations 34 36. As a consequence, only the first quasiparticle band, $E_{1}$, contributes at mean field; for simplicity, we have dropped the band subscript " 1 ". We consider two different sets of solutions, corresponding to the conduction electron filling factors of $n_{c}=0.5$ (which we call set (a)) and $n_{c}=0.8$ (which we call set (b)). We define a Kondo temperature in the lattice for both $\Gamma_{7}$ and $\Gamma_{8}$ multiplets by

$$
T_{o \Gamma} \equiv \epsilon_{\Gamma}-\mu
$$

The reader is reminded that $\mu$ is the quasiparticle chemical potential. The motivation for defining the Kondo temperature as the difference between the shifted multiplet energy $\left(\epsilon_{\Gamma}\right)$ and the quasiparticle chemical potential, is that in the $S U(N)$ model this 
difference has exactly the same structure as the Kondo temperature for the impurity problem. That is, in the $S U(N)$ model, one finds that 31

$$
\epsilon-\mu=D e^{-\left|E_{f}\right| / N N(0) V_{o}^{2}}
$$

where $D$ is the half bandwidth, $N(0)$ is the (assumed flat) conduction electron density of states, $E_{f}$ is the unshifted $4 f$ multiplet energy, and $V_{o}$ is the bare hybridization strength. In table 2, we see that both parameter sets (a) and (b) have approximate Kondo temperatures (for the $\Gamma_{7}$ doublet) of $10 \mathrm{~K}$, which, based on the neutron scattering quasielastic linewidth[21], is a reasonable estimate for $\mathrm{CeCu}_{2} \mathrm{Si}_{2}$. Note, also, that the Kondo temperature for the $\Gamma_{8}$ quartet is dominated by the crystal field splitting,

$$
T_{o 8}=T_{o 7}+\Delta_{C E F} \approx 370 \mathrm{~K}
$$

where $T_{o 7} / T_{o 8} \approx 0.027$. Figure 5 plots the lowest quasiparticle bands for both mean field parameter sets (a) and (b). The top of the first quasiparticle band is just below the shifted $\Gamma_{7}$ energy, $\epsilon_{7}$. The quasiparticle chemical potential cuts through the flat part of the first band in both cases, giving rise to a very large quasiparticle density of states at the Fermi surface.

\section{EFFECTIVE MAGNETIC MOMENT}

In this section we discuss our results for the effective magnetic moment of the quasiparticle states when averaged over the Fermi surface. This calculation was motivated by the work of Zou and Anderson[19], who wished to explain how the small Wilson ratios, $R$, seen in heavy Fermion superconductors (for $\mathrm{CeCu}_{2} \mathrm{Si}_{2}, R \approx 0.5[3]$ ) 
could be reconciled with the presence of strong spin fluctuations. If spin fluctuations are indeed the source of the superconductivity in heavy fermion compounds [32], one would expect a Wilson ratio greater than unity. Zou and Anderson have claimed that, even in the presence of strong spin fluctuations, the Wilson ratio could be reduced due to the anisotropic hybridization between the conduction and the $f$ electrons. They calculated the quasiparticle states, including spin-orbit coupling, from the relativistic KKR equation. Their states are identical to those found via a mean-field approximation to the infinite-U Anderson lattice [11], [13], [33], or via Gutzwiller projection techniques [34].

In spherical symmetry, for a total angular momentum of $J=5 / 2$, the quasiparticle states are

$$
\left|Q_{\vec{k} n \sigma}\right\rangle=A_{n}(|\vec{k}|)\left[|\vec{k} \sigma\rangle-\sum_{m=-5 / 2}^{5 / 2} \frac{s_{o} V_{m \sigma}(\vec{k})}{\left(\epsilon-E_{n k}\right)}|m\rangle\right],
$$

where the normalization for the lowest energy band is

$$
A_{1}^{2}(|\vec{k}|)=\left[1+\frac{s_{o}^{2} V_{o}^{2}}{\left(\epsilon-E_{1 k}\right)^{2}}\right] .
$$

In the preceding equations, $E_{n k}$ is the quasiparticle energy for band $n$ and $\epsilon$ is the energy of the shifted $4 f$ states. Note that the normalization function is isotropic and depends only on the magnitude of the wavevector. For the case of $J=5 / 2$ there is a four-fold degenerate band of energy $\epsilon$, since two of the six total $f$ states have hybridized with the conduction states.

Zou and Anderson calculated the magnetic moment of the quasiparticle states in the lowest band, which we denote as

$$
\left\langle Q_{\vec{k} 1 \sigma}\left|\hat{\mu}_{z}\right| Q_{\vec{k} 1 \sigma}\right\rangle \equiv \mu_{z}^{\sigma \sigma^{\prime}}(\vec{k})
$$


where the magnetic moment operator is

$$
\hat{\mu}_{z}=\hat{l}_{z}+2 \hat{s}_{z}=g_{J} \hat{J}_{z}
$$

$g_{J}$ being the g-factor for the total angular momentum, $J$. The effective magnetic moment is the average of $\mu_{z}^{\sigma \sigma^{\prime}}$ over the spherical Fermi surface,

$$
\mu_{e f f}^{2}=\int \frac{d \hat{k}}{4 \pi}\left[\mu_{z}^{+1+1}(\vec{k})^{2}+\mu_{z}^{+1-1}(\vec{k})^{2}\right]
$$

and the result is $\mu_{\text {eff }}^{2}=1.16 \mu_{B}^{2}$, where $\mu_{B}$ is the Bohr-magneton. Note that the bare moment for a $f^{1}$ state is $\mu=2.54 \mu_{B}$, so that hybridization has reduced the size of the moment.

The Pauli susceptibility for the quasiparticles is a function of the effective magnetic moment and the renormalized density of states at the Fermi surface, $\tilde{N}(0)(\tilde{N}(0)=$ $\frac{m^{*}}{m} N(0)$, where $m^{*}$ is the quasiparticle effective mass),

$$
\chi_{\text {Pauli }}=2 \mu_{\text {eff }}^{2} \tilde{N}(0) .
$$

Substituting this value for the Pauli susceptibility into the expression for the Wilson ratio gives

$$
R_{\text {reduced }}=\left(\frac{\mu_{e f f}}{\mu}\right)^{2} \frac{1}{1+F_{0}^{a}}=\frac{0.18}{1+F_{0}^{a}} .
$$

In this way, it would be possible to have a large Stoner factor, $\left(1+F_{0}^{a}\right)^{-1}$, as befits the presence of strong spin fluctuations, but the reduced magnetic moment could still account for a small experimentally observed Wilson ratio.

Cox 35, Zhang and Lee [36], and Aeppli and Varma 37] pointed out the need to include the Van Vleck susceptibility in the calculation. The value for $\chi$ in equation 46 must be the total susceptibility, as seen by experiment. In the case of the uniform 
susceptibility, the Van Vleck contribution represents direct transitions from the Fermi energy in the lowest band to higher energy bands. The dominant contribution must come from transitions to the four-fold degenerate band lying at an energy $T_{o}$ above the Fermi energy, where $T_{o}$ is the Kondo temperature in spherical symmetry. The Van Vleck susceptibility then has the structure

$$
\chi_{V V}(\vec{q}=0)=2 \mu_{B}^{2} \sum_{m} \int \frac{d \hat{k}}{4 \pi} \frac{\left|\left\langle Q_{\vec{k} 1 \sigma}\left|\hat{\mu}_{z}\right| m\right\rangle\right|^{2}}{T_{o}}
$$

where the quasiparticle density of states $\tilde{N}(0) \approx 1 / T_{o}$. The small energy denominator (for $\mathrm{CeCu}_{2} \mathrm{Si}_{2}, T_{o} \approx 1 \mathrm{meV}$ ) makes this interband contribution to the susceptibility comparable to the contribution from the Pauli susceptibility. Cox and Zhang and Lee showed that the total susceptibility was given by

$$
\chi_{\text {total }}=\chi_{\text {Pauli }}+\chi_{V V}=\frac{1}{3} g_{J}^{2} J(J+1) \mu_{B}^{2}[2 \tilde{N}(0)]
$$

which depends on the bare magnetic moment. Thus the inclusion of the Van Vleck susceptibility would alter Zou and Anderson's argument.

We now wish to discuss what happens when crystal electric fields of cubic symmetry are included. The quasiparticle states are given by equation 22, where the local crystal field states are orthonormal to each other. Note that in this case the dispersionless band lies at an energy $\Delta_{C E F}$ above the Fermi energy, where the crystal field splitting is much larger than the Kondo temperature. In order to calculate the effective magnetic moment, we need the expectation value of equation 42 for the lowest quasiparticle band. We find that we can write equation 42 in the form

$$
\mu_{z}^{\sigma \sigma^{\prime}}(\vec{k})=A_{1}^{2}(\vec{k})\left[\sigma \delta_{\sigma \sigma^{\prime}}+\sum_{\Gamma^{\prime} \alpha^{\prime} \Gamma \alpha m} \frac{m g_{J} c_{\Gamma^{\prime} \alpha^{\prime} m} s_{o \Gamma^{\prime}} V_{\Gamma^{\prime} \alpha^{\prime} \sigma}^{*}(\vec{k}) V_{\Gamma \alpha \sigma^{\prime}}(\vec{k}) s_{o \Gamma} c_{\Gamma \alpha m}}{\left(\epsilon_{\Gamma^{\prime}}-E_{1 \vec{k}}\right)\left(\epsilon_{\Gamma}-E_{1 \vec{k}}\right)}\right] .
$$


Using equation 44, we find the average moment is

$$
\mu_{\text {cubic,eff }}^{2}=0.583 \mu_{B}^{2}
$$

for mean-field parameter set (a). For a free $\Gamma_{7}$ doublet the average moment is

$$
\mu_{7}^{2}=\frac{25}{49} \mu_{B}^{2}
$$

The effective magnetic moment in cubic symmetry is slightly larger than the value for a free $\Gamma_{7}$ moment. This was expected by Cox 35], who predicted the effective moment would have the structure

$$
\mu_{e f f}^{2}=\mu_{7}^{2}\left[1+\alpha \sqrt{\frac{m}{m^{*}}}\right]
$$

where $\alpha$ is a prefactor that could be as big as about 10. The contributions of order $\sqrt{m / m^{*}}$ come from the six regions on the Fermi surface which intersect the axes of the cubic Brillouin zone. These are the so-called "hot-spots", which we have already mentioned. At these points on the Fermi surface, the plane wave conduction states and the localized $\Gamma_{7}$ states can not hybridize. Thus we expect the $g$-factor at these "spots" to go back to the free electron value of 2 , with the result that the contribution to $\mu_{\text {eff }}$ from the "hot-spots" increases the effective moment above that of a free moment of $\Gamma_{7}$ symmetry. For parameter set (a), we have

$$
\frac{m}{m^{*}}=\left(\frac{T_{o 7}}{s_{o} V_{o}}\right)^{2}=1.33 \times 10^{-4} .
$$

Equation 52 is applicable for the quoted values of $\mu_{\text {eff }}^{2}$ and $\mu_{7}^{2}$ if $\alpha=9.23$, which is a reasonable value.

Finally, we substitute $\mu_{e f f}^{2}$ into the expression for the Wilson ratio,

$$
R=\left(\frac{\pi k_{B}}{\mu_{e f f}}\right)^{2} \frac{\chi(0)}{\gamma(0)}
$$


where $\chi(0)$ is the low temperature susceptibility, $\gamma(0)$ is the linear coefficient of specific heat, $k_{B}$ is Boltzmann's constant, and $\mu_{B}$ is the Bohr magneton. Using $\chi(0)=0.019$ $\mathrm{emu} / \mathrm{mole}$ (for a magnetic field along the c axis of the tetragonal unit cell) [38], and $\gamma(0)=1000 \mathrm{~mJ} / \mathrm{mole}-\mathrm{K}^{2}$ [回 gives $R=2.38$. It is possible that experimentally quoted results of approximately 0.5 for the Wilson ratio [3] are too small since the bare effective moment of $\mu_{\text {eff }}^{2}=2.54 \mu_{B}^{2}$ was used in equation 52. We also find it interesting to note that for the two-channel Kondo impurity, the Wilson ratio is approximately 2.6 .

\section{HYBRIDIZATION DRESSED PROPAGATORS}

With the eigenstates of the mean field Hamiltonian written in the undiagonalized basis, there are three fermionic Green functions: $\mathrm{G}_{\Gamma \alpha \Gamma^{\prime} \alpha^{\prime}}$ (the f Green function); $\mathrm{G}_{\sigma}$ (the conduction Green function); and $\mathrm{G}_{\Gamma \alpha \sigma}$ (the off-diagonal, or mixing, Green function). All three are defined below in terms of Fock space operators:

$$
\begin{aligned}
G_{\Gamma \alpha \Gamma^{\prime} \alpha^{\prime}}(\vec{k}, \tau) & \equiv-\left\langle T_{\tau} f_{\vec{k} \Gamma \alpha}(\tau) f_{\vec{k} \Gamma^{\prime} \alpha^{\prime}}^{\dagger}(0)\right\rangle, \\
G_{\sigma}(\vec{k}, \tau) & \equiv-\left\langle T_{\tau} c_{\vec{k} \sigma}(\tau) c_{\vec{k} \sigma}^{\dagger}(0)\right\rangle, \\
G_{\Gamma \alpha \sigma}(\vec{k}, \tau) & \equiv-\left\langle T_{\tau} f_{\vec{k} \Gamma \alpha}(\tau) c_{\vec{k} \sigma}^{\dagger}(0)\right\rangle,
\end{aligned}
$$

where $T_{\tau}$ is the imaginary time ordering operator. It is easy to see, diagrammatically, how these hybridization dressed Green functions can be calculated. Figures 6,7, and 8 show the expansions for $G_{\Gamma \alpha \Gamma^{\prime} \alpha^{\prime}}, G_{\sigma}$, and $G_{\Gamma \alpha \sigma}$, respectively. In terms of a complex 
frequency, $z$, the mean-field Green functions are:

$$
\begin{gathered}
G_{\Gamma \alpha \Gamma^{\prime} \alpha^{\prime}}(\vec{k}, z)=\frac{1}{z-\epsilon_{\Gamma}}\left[\delta_{\Gamma \Gamma^{\prime}} \delta_{\alpha \alpha^{\prime}}+\frac{1}{z-\epsilon_{\Gamma^{\prime}}} \frac{\left(z-\epsilon_{7}\right)\left(z-\epsilon_{8}\right) \tilde{s}_{o \Gamma} \tilde{V}_{\Gamma \alpha \sigma}^{*}(\vec{k}) \tilde{V}_{\Gamma^{\prime} \alpha^{\prime} \sigma}(\vec{k}) \tilde{s}_{o \Gamma^{\prime}}}{\left(z-E_{1 \vec{k}}\right)\left(z-E_{2 \vec{k}}\right)\left(z-E_{3 \vec{k}}\right)}\right], \\
G_{\sigma}(\vec{k}, z)=\frac{\left(z-\epsilon_{7}\right)\left(z-\epsilon_{8}\right)}{\left(z-E_{1 \vec{k}}\right)\left(z-E_{2 \vec{k}}\right)\left(z-E_{3 \vec{k}}\right)}, \\
G_{\Gamma \alpha \sigma}(\vec{k}, z)=\frac{\tilde{s}_{o \Gamma} \tilde{V}_{\Gamma \alpha \sigma}^{*}(\vec{k})\left(z-\epsilon_{\Gamma^{*}}\right)}{\left(z-E_{1 \vec{k}}\right)\left(z-E_{2 \vec{k}}\right)\left(z-E_{3 \vec{k}}\right)} .
\end{gathered}
$$

These propagators will be used to calculate the dressed bosonic Green functions.

\section{FLUCTUATIONS}

In this section, we shall calculate the Green functions for the Bosonic fields in our Hamiltonian, namely $\tilde{s}_{\vec{k} \Gamma}$ and $i \lambda_{\vec{k}}$. The Bosonic Green functions can be dressed (through the terms $H_{m i x}$ and $H_{f}$ ) by particle-hole excitations of the hybridized conduction and $4 f$ electron systems. To proceed, we write the slave Boson and Lagrange multiplier as follows:

$$
\begin{aligned}
& \tilde{s}_{\vec{k} \Gamma}=\tilde{s}_{o \Gamma} \delta_{\vec{k}, 0}+\delta \tilde{s}_{\vec{k} \Gamma}, \\
& i \lambda_{\vec{k}}=i \lambda_{o} \delta_{\vec{k}, 0}+i \delta \lambda_{\vec{k}},
\end{aligned}
$$

where $\delta \tilde{s}_{\vec{k} \Gamma}$ and $\delta \lambda_{\vec{k}}$ represent fluctuations away from the (self-consistent) mean field values.

It is easy to see how the particle-hole excitations dress the Bosonic Green functions by writing the full Hamiltonian in k-space,

$$
H=\sum_{\vec{k} \sigma} \xi_{\vec{k}} c_{\vec{k} \sigma}^{\dagger} c_{\vec{k} \sigma}+\sum_{\vec{k} \vec{k}^{\prime} \Gamma \alpha} f_{\vec{k} \Gamma \alpha}^{\dagger}\left[E_{\Gamma} \delta_{\vec{k} \vec{k}^{\prime}}+i \lambda_{\vec{k}-\vec{k}^{\prime}}\right] f_{\vec{k}^{\prime} \Gamma \alpha}
$$




$$
\begin{aligned}
& +\sum_{\vec{k} \vec{k}^{\prime} \Gamma \alpha \sigma}\left[\tilde{V}_{\Gamma \alpha \sigma}(\vec{k}) c_{\vec{k} \sigma}^{\dagger} f_{\vec{k}^{\prime} \Gamma \alpha} \tilde{s}_{\vec{k}^{\prime}-\vec{k} \Gamma}^{*}+H . c .\right] \\
& +\frac{N_{s}}{2} \sum_{\vec{k} \Gamma} N_{\Gamma} i \lambda_{\vec{k}}\left[\sum_{\vec{k}^{\prime}} \tilde{s}_{\vec{k}+\vec{k}^{\prime} \Gamma}^{*} \tilde{s}_{\vec{k} \Gamma}-q_{\Gamma}\right] .
\end{aligned}
$$

Substituting from equations 60 and 61 , equation 62 can be written in two pieces, one representing the mean field approximation (which we have solved), and the second piece coming from the fluctuations in the Bosonic fields, $\delta \tilde{s}_{\vec{k} \Gamma}$ and $\delta \lambda_{\vec{k}}$. The bare Boson Green functions come from the terms in the constraint, the last line of equation 62, which are quadratic in the fluctuating fields. Since this involves terms of the form $\delta \tilde{s}_{\vec{k} \Gamma} \delta \tilde{s}_{\vec{k} \Gamma}, \delta \tilde{s}_{\vec{k} \Gamma} \delta \lambda_{\vec{k}}$, and $\delta \lambda_{\vec{k}} \delta \lambda_{\vec{k}}$, we write the bare propagator in a matrix form (in the static limit),

$$
\hat{D}_{o \Gamma \Gamma^{\prime}}^{-1}=-\frac{N_{\Gamma}}{2}\left(\begin{array}{cc}
i \lambda_{o} & \tilde{s}_{o \Gamma} \\
\tilde{s}_{o \Gamma} & 0
\end{array}\right) \delta_{\Gamma \Gamma^{\prime}}
$$

where $\tilde{s}_{o \Gamma}=s_{o} / \sqrt{N_{\Gamma}}$. The specific elements of the matrix are $D_{o s s \Gamma \Gamma^{\prime}}=-\frac{N_{\Gamma}}{2} i \lambda_{o} \delta_{\Gamma \Gamma^{\prime}}$, $D_{o s \lambda \Gamma \Gamma^{\prime}}=-\frac{N_{\Gamma}}{2} \tilde{s}_{o \Gamma} \delta_{\Gamma \Gamma^{\prime}}$, and $D_{o \lambda \lambda \Gamma \Gamma^{\prime}}=0$.

The dressed Boson Green function will then satisfy a matrix Dyson's equation,

$$
\hat{D}_{\Gamma \Gamma^{\prime}}^{-1}(\vec{q})=\hat{D}_{o \Gamma \Gamma^{\prime}}^{-1}(\vec{q})-\hat{\Pi}_{\Gamma \Gamma^{\prime}}(\vec{q})
$$

The $2 \times 2$ self-energy matrix, $\hat{\Pi}_{\Gamma^{\prime}}(\vec{q})$, due to the particle-hole excitations, can be calculated by the usual Feynman diagrammatic techniques.

To motivate the results for $\hat{D}_{\Gamma \Gamma^{\prime}}^{-1}$, consider the diagonal component of the matrix Dyson's equation in Figure 9(a). Taking all contributions to the self-energy of order $N_{\Gamma}$ would give a dressed propagator of order $1 / N_{\Gamma}$. In Figure $9(\mathrm{c})$, the $\times$ symbols at the corners of the diagrams represent scaled hybridization matrix elements, $\tilde{V}_{\Gamma \alpha \sigma}(\vec{k})$, which are of order one $(\mathcal{O}(1))$. Thus, since there is a sum over the degenerate states 
$\alpha$ of the multiplet $\Gamma$ and no factor of $1 / N_{\Gamma}$ from the matrix elements to cancel it, we say that the Boson self-energy diagram is of order $N_{\Gamma}$. Technically, there is not a free sum over the degeneracy label $\alpha$ of a given multiplet, because the hybridization matrix elements, $\tilde{V}_{\Gamma \alpha \sigma}(\vec{k})$, are dependent upon $\alpha$. (That is, we do not have an explicit factor of $N_{\Gamma}$ after summing over $\alpha$.) In the $S U(N)$ model, the isotropic hybridization matrix element, $V_{o}$, is truly independent of the degeneracy label, m. Thus the sum over $m$ yields a factor of $N$. In our case, even though we do not have explicit factors of $N_{\Gamma}$, we assume that the closed Fermionic bubbles are the important diagrams, in analogy with the $S U(N)$ model.

Figure 9 shows all the unique self-energy diagrams in terms of the three hybridizationdressed Fermionic Green functions, equations 5456. Evaluation of theses diagrams leads to the following results for the elements of the (inverse) dressed Bose propagator:

$$
\begin{gathered}
D_{s s \Gamma \Gamma^{\prime}}^{-1}(\vec{q})=2 I_{s s \Gamma \Gamma^{\prime}}(\vec{q}) \\
D_{s \lambda \Gamma \Gamma^{\prime}}^{-1}=-i\left[\frac{N_{\Gamma}}{2} \tilde{s}_{o \Gamma}+\frac{x_{\Gamma}}{\tilde{s}_{o \Gamma}}\right] \delta_{\Gamma \Gamma^{\prime}}+i I_{s \lambda \Gamma \Gamma^{\prime}}(\vec{q}), \\
D_{\lambda \lambda \Gamma \Gamma^{\prime}}^{-1}(\vec{q})=-\frac{y_{\Gamma}}{T_{o \Gamma}}-\frac{1}{2} I_{\lambda \lambda \Gamma \Gamma^{\prime}}(\vec{q}),
\end{gathered}
$$

where the momentum dependent functions are given by

$$
\begin{gathered}
I_{s s \Gamma \Gamma^{\prime}}(\vec{q}) \equiv \frac{P}{N_{s}} \sum_{\vec{k} \vec{k}^{\prime} \alpha \alpha^{\prime}} \frac{f\left(E_{1 \vec{k}}\right) \tilde{s}_{O \Gamma} \tilde{s}_{O \Gamma^{\prime}} \mu_{\Gamma \alpha \Gamma^{\prime} \alpha^{\prime}}(\vec{k}) \mu_{\Gamma^{\prime} \alpha^{\prime} \Gamma \alpha}\left(\vec{k}^{\prime}\right)\left(\epsilon_{7}-E_{1 \vec{k}}\right)^{2}\left(\epsilon_{8}-E_{1 \vec{k}}\right)^{2}}{\left(E_{1 \vec{k}^{\prime}}-E_{1 \vec{k}}\right)\left(E_{2 \vec{k}^{\prime}}-E_{1 \vec{k}}\right)\left(E_{3 \vec{k}^{\prime}}-E_{1 \vec{k}}\right)\left(E_{2 \vec{k}}-E_{1 \vec{k}}\right)} \\
\quad \times \frac{\left[\delta_{\vec{k}^{\prime}, \vec{k}+\vec{q}}+\delta_{\vec{k}^{\prime}, \vec{k}-\vec{q}}\right]}{\left(\epsilon_{\Gamma}-E_{1 \vec{k}}\right)\left(\epsilon_{\Gamma^{\prime}}-E_{1 \vec{k}}\right)\left(E_{3 \vec{k}}-E_{1 \vec{k}}\right)}, \\
I_{s \lambda \Gamma \Gamma^{\prime}}(\vec{q}) \equiv \frac{P}{N_{s}} \sum_{\vec{k} \vec{k}^{\prime}} \frac{f\left(E_{1 \vec{k}}\right)\left(\epsilon_{7}-E_{1 \vec{k}}\right)^{2}\left(\epsilon_{8}-E_{1 \vec{k}}\right)^{2}}{\left(E_{2 \vec{k}}-E_{1 \vec{k}}\right)\left(E_{3 \vec{k}}-E_{1 \vec{k}}\right)\left(E_{1 \vec{k}^{\prime}}-E_{1 \vec{k}}\right)\left(E_{2 \vec{k}^{\prime}}-E_{1 \vec{k}}\right)\left(E_{3 \vec{k}^{\prime}}-E_{1 \vec{k}}\right)}
\end{gathered}
$$




$$
\begin{gathered}
\times \sum_{\alpha \alpha^{\prime}} \frac{\tilde{s}_{o \Gamma} \tilde{s}_{o \Gamma^{\prime}}}{\left(E_{1 \vec{k}}-\epsilon_{\Gamma}\right)\left(E_{1 \vec{k}}-\epsilon_{\Gamma^{\prime}}\right)}\left[\frac{\tilde{s}_{o \Gamma} \mu_{\Gamma \alpha \Gamma^{\prime} \alpha^{\prime}}(\vec{k}) \mu_{\Gamma^{\prime} \alpha^{\prime} \Gamma \alpha}\left(\vec{k}^{\prime}\right)}{\left(E_{1 \vec{k}}-\epsilon_{\Gamma}\right)}\right. \\
\left.+\frac{\tilde{s}_{o \Gamma^{\prime}} \mu_{\Gamma^{\prime} \alpha^{\prime} \Gamma \alpha}(\vec{k}) \mu_{\Gamma \alpha \Gamma^{\prime} \alpha^{\prime}}\left(\vec{k}^{\prime}\right)}{\left(E_{1 \vec{k}}-\epsilon_{\Gamma^{\prime}}\right)}\right]\left[\delta_{\vec{k}^{\prime}, \vec{k}+\vec{q}}+\delta_{\overrightarrow{k^{\prime}}, \vec{k}-\vec{q}}\right], \\
I_{\lambda \lambda \Gamma \Gamma^{\prime}}(\vec{q}) \equiv \frac{P}{N_{s}} \sum_{\vec{k} \vec{k}^{\prime} \alpha \alpha^{\prime}} \frac{f\left(E_{1 \vec{k}}\right) \tilde{s}_{o \Gamma}^{2} \tilde{s}_{o \Gamma^{\prime}}^{2} \mu_{\Gamma \alpha \Gamma^{\prime} \alpha^{\prime}}\left(\vec{k}^{\prime}\right) \mu_{\Gamma^{\prime} \alpha^{\prime} \Gamma \alpha}(\vec{k})\left[\delta_{\vec{k}^{\prime}, \vec{k}-\vec{q}}+\delta_{\vec{k}^{\prime}, \vec{k}+\vec{q}}\right]}{\left(E_{1 \vec{k}^{\prime}}-E_{1 \vec{k}}\right)\left(E_{2 \vec{k}^{\prime}}-E_{1 \vec{k}}\right)\left(E_{3 \vec{k}^{\prime}}-E_{1 \vec{k}}\right)\left(E_{2 \vec{k}}-E_{1 \vec{k}}\right)} \\
\quad \times \frac{\left(\epsilon_{7}-E_{1 \vec{k}}\right)^{2}\left(\epsilon_{8}-E_{1 \vec{k}}\right)^{2}}{\left(\epsilon_{\Gamma}-E_{1 \vec{k}}\right)^{2}\left(\epsilon_{\Gamma^{\prime}}-E_{1 \vec{k}}\right)^{2}\left(E_{3 \vec{k}}-E_{1 \vec{k}}\right)} .
\end{gathered}
$$

In equations 68 - 70, $E_{n \vec{k}}$ are the quasiparticle band energies. The quasiparticle chemical potential was chosen to lie in the lowest band, such that $f\left(E_{n \vec{k}}\right)$ is nonzero only for $n=1$. (Recall this is zero temperature calculation.) The anisotropic function $\mu_{\Gamma \alpha \Gamma^{\prime} \alpha^{\prime}}(\vec{k})$ is defined in equation 24. The parameters $x_{\Gamma}$ and $y_{\Gamma}$ are numbers which depend on the mean field parameter set used. See Table 3 for the values of these parameters.

Note the presence of the principal value integrals over the Brillouin zone. We are forced to evaluate the integrals numerically, as discussed in the next section. This is the most labor-intensive part of the calculation.

\section{ANALYTIC TETRAHEDRON METHOD}

We wish to evaluate the three dimensional principal value integrals that have arisen in the calculation of the Bosonic self-energy, i.e. the functions $I_{s s \Gamma \Gamma^{\prime}}(\vec{q})$, $I_{s \lambda \Gamma \Gamma^{\prime}}(\vec{q})$, and $I_{\lambda \lambda \Gamma \Gamma^{\prime}}(\vec{q})$ of equations 68-70. To do so, we use a procedure developed

originally to calculate the real part of spectral functions of the form 39, 40, 41, 44,

$$
\Phi^{\prime}(E)=\operatorname{Re} \Phi(E)=P \frac{1}{N_{s}} \sum_{\vec{k}} \frac{M(\vec{k})}{E-E_{n \vec{k}}} .
$$


$\Phi^{\prime}$ looks like the real part of a dynamic susceptibility. Handled numerically, the principal value nature of the sum in equation 71 makes it non-trivial. In fact, if we tried to do the sum by simply evaluating the function at many points throughout the zone, and then multiplying by a weighting factor, we would find an essentially infinite variance. That is, upon averaging the result over many different mesh sizes, the variance of the mean would be huge compared to the mean-value itself 43 .

The analytic tetrahedron method 41, 42 evaluates expressions like that of equation 71 by breaking up the Brillouin zone into tetrahedra, where the band energies $E_{n \vec{k}}$ need to be known only at the four corners of a given tetrahedron. Any band energy for $\vec{k}$ inside a tetrahedron is interpolated from the energies at the corners. Within this assumption of linear interpolation, and also assuming the effective matrix element, $M(\vec{k})$, is constant inside the tetrahedron, Rath and Freeman 41], and independently Lingård [42] showed that the principal value integral over the tetrahedron could be performed analytically.

We write our functions $I_{a b Г \Gamma^{\prime}}(\vec{q})$ in the following form:

$$
I_{a b \Gamma \Gamma^{\prime}}(\vec{q})=\frac{P}{N_{s}} \sum_{\vec{k} \vec{k}^{\prime}} \frac{f\left(E_{1 \vec{k}}\right) M_{a b \Gamma \Gamma^{\prime}}\left(\vec{k}, \vec{k}^{\prime}\right)}{E_{1 \vec{k}^{\prime}}-E_{1 \vec{k}}} \delta_{\vec{k}^{\prime}, \vec{k} \pm \vec{q}},
$$

where the matrix elements are a complicated function of $\vec{k}$ and $\vec{q}$ which come from equations 68, 69, and 70. That is, direct comparison of equation 72 with equations 68 70 gives the structure of the matrix elements $M_{a b \Gamma \Gamma^{\prime}} \vec{k}, \vec{k}^{\prime}$. Note that $a b$ can represent $s s, s \lambda$, or $\lambda \lambda$. The Fermi function, of course, restricts us to only the volume below the Fermi surface. Since the surfaces of constant energy are planar, we need to know the possible unique ways a plane can cut through a tetrahedron. It turns out, fortunately, that there are only three such ways; and furthermore, in each of the three 
cases, the two subdivided volumes of the intersected tetrahedron (the volume above the Fermi surface and the volume below the Fermi surface) are themselves either a single tetrahedron or a composite of three tetrahedra. We never deal with anything but tetrahedra. So it is just a matter of geometry to find out which tetrahedra are beneath the Fermi surface and hence contribute to the integral of equation 72 .

To evaluate our self-energies, we need the analytic result for the following integral over a tetrahedron:

$$
I=\int_{\text {tetra }} \frac{d^{3} k}{E_{1 \vec{k}^{\prime}}-E_{1 \vec{k}}}
$$

where $\vec{k}^{\prime}=\vec{k} \pm \vec{q}$. For simplicity of notation we define the energy differences

$$
V_{i} \equiv E_{1 \vec{k}^{\prime} i}-E_{1 \vec{k} i},
$$

where the "1" signifies the band index, and $i=1,2,3,4$ labels the four corners of the tetrahedron. The result for the integral $I$ depends only on the $V_{i}$ and the volume of the tetrahedron. Using the notation of Rath and Freeman[41] the results is:

$$
I=3 v_{\text {tet }}\left(\frac{V_{1}^{2}}{D_{1}} \ln \left|\frac{V_{1}}{V_{4}}\right|+\frac{V_{2}^{2}}{D_{2}} \ln \left|\frac{V_{2}}{V_{4}}\right|+\frac{V_{3}^{2}}{D_{3}} \ln \left|\frac{V_{3}}{V_{4}}\right|\right),
$$

where $v_{\text {tet }}$ is the volume of the tetrahedron, and where

$$
\begin{aligned}
& D_{1}=\left(V_{1}-V_{4}\right)\left(V_{1}-V_{3}\right)\left(V_{1}-V_{2}\right) \\
& D_{2}=\left(V_{2}-V_{4}\right)\left(V_{2}-V_{3}\right)\left(V_{2}-V_{1}\right) \\
& D_{3}=\left(V_{3}-V_{4}\right)\left(V_{3}-V_{2}\right)\left(V_{3}-V_{1}\right) .
\end{aligned}
$$

We have studied equation 75 carefully in the limits where some of the $V_{i}$ s are equal to each other or are equal to zero. It turns out that there are quite a few such cases, but for the sake of space, we do not tabulate them here[44]. 
Note that these three dimensional integrals can have an entire surface of poles inside the Brillouin zone that must be handled properly. In contrast, a one-dimensional principal value integral can be regularized numerically by basically subtracting off the divergence 45, and such a procedure is facilitated by the relatively small number of poles throughout the domain of integration. In our three-dimensional integral, if $n_{\text {mesh }}^{3}$ is the number of sub-cubes inside the cubic Brillouin zone, then there are of the order of $n_{m e s h}^{2}$ poles, which clearly gets large as $n_{m e s h}$ increases, and it is no longer possible to regularize the integral in a simple way. The large quantity of work done by (mostly electronic structure) physicists in this field of numerical k-space sums, is indicative of the degree of complexity inherent to these problems [46], 477.

We now show the results for the self-energy functions $I_{s s}(\vec{q}), I_{s \lambda}(\vec{q})$, and $I_{\lambda \lambda}(\vec{q})$, which have been summed over the crystal field multiplet indices,

$$
\begin{gathered}
I_{s s}(\vec{q}) \equiv \sum_{\Gamma \Gamma^{\prime}} \frac{I_{s s \Gamma \Gamma^{\prime}}(\vec{q})}{\sqrt{N_{\Gamma}} \sqrt{N_{\Gamma^{\prime}}}}, \\
I_{s \lambda}(\vec{q})=\sum_{\Gamma \Gamma^{\prime}}\left(\frac{I_{s \lambda \Gamma \Gamma^{\prime}}(\vec{q})}{\sqrt{N_{\Gamma}}}+\frac{I_{s \lambda \Gamma \Gamma^{\prime}}(\vec{q})}{\sqrt{N_{\Gamma^{\prime}}}}\right),
\end{gathered}
$$

and

$$
I_{\lambda \lambda}(\vec{q})=\sum_{\Gamma \Gamma^{\prime}} I_{\lambda \lambda \Gamma \Gamma^{\prime}}(\vec{q})
$$

For a given mesh parameter, $n_{m e s h}$, the total number of tetrahedra in the Brillouin zone is $8 \times n_{\text {mesh }}^{3}$. The functions $I_{s s}, I_{s \lambda}$, and $I_{\lambda \lambda}$ are plotted as a function of the mesh parameter, $n_{m e s h}$, in Figures 10-12 for mean field parameter set (a). The results for parameter set (b) are similar. We chose the momentum $q=0.5 \hat{z}$, measured in units of $\pi / a$, where $a$ is the lattice spacing. (We take $a=3.89 \AA$.) The matrix elements are assumed constant inside a given tetrahedron. Since they are complicated anisotropic 
functions, however, the matrix elements can vary a great deal throughout the Brillouin zone, and thus they cause fluctuations in the value of the $I_{a b}(\vec{q})$ as a function of $n_{\text {mesh }}$. This is clearly visible in figures $10-12$.

It is clear that if we need convergence of the Green functions to several decimal places, it would require a mesh with $n_{\text {mesh }}>80$. In background, a calculation for $n_{\text {mesh }}=40$ takes about one hour on a DEC 5100. Since the computing time goes as $n_{\text {mesh }}^{3}$, it is clear that a run with $n_{\text {mesh }}=80$ requires a long run time. Attempts at vectorization of the code were hindered by a plethora of logic statements required for the subroutine that performed the integral in equation 73. Actually, this numerical integration is parallel in nature. Each of the $n_{\text {mesh }}^{3}$ subcubes could, in principle, be integrated independently of the others, and at the end the net result would be the sum of the results from each subcube. Naively, this is the kind of problem a parallel machine should be able to handle well.

It is also interesting to note that calculations of the susceptibility or dielectric constant based on electronic structure data, generally are not performed for $n_{\text {mesh }}$ greater than about 30 [48]. The complicated nature of our matrix elements have forced us to push the procedure to very large values (by anyone's standards) of $n_{m e s h}$. We shall discuss in the next section, how our conclusions on pairing instabilities in the infinite-U Anderson lattice take into account this slow convergence of the Bosonic self-energy.

\section{QUASIPARTICLE SCATTERING AMPLITUDE}

In this section, we present our analysis of the scattering amplitude $\Gamma_{Q P}\left(\vec{k}, \vec{k}^{\prime}\right)$, 
which allows for quasiparticle interactions via the exchange of $4 \mathrm{f}$ density fluctuations. The exchanged Boson will be represented by the (dressed to order 1/N) Boson matrix Green function calculated in Sec. VIII. The diagrams for the scattering amplitude are presented in Figure 13, where the straight lines represent the incoming (and outgoing) quasiparticles and the wavy line is an element of the (matrix) Boson Green function, $\hat{D}_{\Gamma \Gamma^{\prime}}(\vec{q})$. The large black circles represent quasiparticle vertices, $\gamma_{f \Gamma}\left(\vec{k}, \vec{k}^{\prime}\right)$ and $\gamma_{m i x \Gamma}\left(\vec{k}, \vec{k}^{\prime}\right)$, which are calculated by writing the full Hamiltonian in the quasiparticle basis:

$$
\begin{gathered}
H_{Q P}=\sum_{\vec{k} \sigma n n^{\prime}} \xi_{\vec{k}} A_{n}^{*}(\vec{k}) A_{n^{\prime}}(\vec{k}) Q_{\vec{k} n \sigma}^{\dagger} Q_{\vec{k} n^{\prime} \sigma} \\
+\sum_{\vec{k} \vec{k}^{\prime} \Gamma \alpha n n^{\prime} \sigma \sigma^{\prime}} \gamma_{f \Gamma}\left(\vec{k}, \vec{k}^{\prime}\right) Q_{\vec{k} n \sigma}^{\dagger}\left[E_{\Gamma} \delta_{\vec{k} \vec{k}^{\prime}}+i \lambda_{\vec{k}-\vec{k}^{\prime}}\right] Q_{\vec{k}^{\prime} n^{\prime} \sigma^{\prime}} \\
+\sum_{\vec{k} \vec{k}^{\prime} \Gamma \alpha \sigma n n^{\prime} \sigma^{\prime}}\left[\gamma_{m i x \Gamma}\left(\vec{k}, \vec{k}^{\prime}\right) Q_{\vec{k} n \sigma}^{\dagger} Q_{\vec{k}^{\prime} n^{\prime} \sigma^{\prime}} \tilde{s}_{\overrightarrow{k^{\prime}}-\vec{k} \Gamma}^{*}+H . c .\right]+H_{\text {constraint }}
\end{gathered}
$$

Recall that $Q_{\vec{k} n \sigma}$ destroys a quasiparticle of momentum $\vec{k}$, pseudospin $\sigma$, and band index $n$. The vertex functions $\gamma_{f}$ and $\gamma_{\text {mix }}$ thus come from the unitary transformation that diagonalizes $H_{M F}$ (see equations 26 and 27)

$$
\begin{aligned}
\gamma_{f \Gamma}\left(\vec{k}, \vec{k}^{\prime}\right) & \equiv \frac{A_{n}^{*}(\vec{k}) A_{n^{\prime}}\left(\vec{k}^{\prime}\right) \tilde{s}_{o \Gamma}^{2} \tilde{V}_{\Gamma \alpha \sigma^{\prime}}^{*}\left(\vec{k}^{\prime}\right) \tilde{V}_{\sigma \Gamma \alpha}(\vec{k})}{\left(\epsilon_{\Gamma}-E_{n \vec{k}}\right)\left(\epsilon_{\Gamma}-E_{n^{\prime} \vec{k}^{\prime}}\right)}, \\
\gamma_{m i x \Gamma}\left(\vec{k}, \vec{k}^{\prime}\right) & \equiv-\frac{A_{n}^{*}(\vec{k}) A_{n^{\prime}}\left(\vec{k}^{\prime}\right) \tilde{s}_{o \Gamma} \tilde{V}_{\Gamma \alpha \sigma^{\prime}}^{*}\left(\vec{k}^{\prime}\right) \tilde{V}_{\Gamma \alpha \sigma}(\vec{k})}{\epsilon_{\Gamma}-E_{n^{\prime} \vec{k}^{\prime}}} .
\end{aligned}
$$

As shown below, we project $\Gamma_{Q P}\left(\vec{k}, \vec{k}^{\prime}\right)$ onto states of cubic symmetry, $\Phi_{\eta}(\vec{k})$, the so-called cubic harmonics, where $\eta$ labels the irreducible representations of the octahedral group $\mathcal{O}_{h}$. The product is then averaged over the Fermi surface,

$$
\Gamma_{\eta}=\int \frac{d \hat{k}}{4 \pi} \int \frac{d \hat{k}^{\prime}}{4 \pi} \Phi_{\eta}^{*}\left(\hat{k}^{\prime}\right) \Gamma_{Q P}\left(\vec{k}, \vec{k}^{\prime}\right) \Phi_{\eta}(\hat{k}) .
$$


A superconducting instability of symmetry $\eta$ is signaled by a negative value of the corresponding average, $\Gamma_{\eta}$. See Table 4 for a list of the cubic harmonics used in this calculation. The character table for the octahedral group $\mathcal{O}$ is presented in Table 5, where the irreducible representations are listed: $A_{1}, A_{2}, E, T_{1}$, and $T_{2}$. The group $\mathcal{O}_{h}$ follows from the group $\mathcal{O}$ by including inversions. This means the representations pick up a subscript " $g$ " or " $u$ " depending on if the they are even or odd, respectively, under parity. Because of the complexity in calculating the dressed bose propagators we have assumed a spherical Fermi surface for the average in equation 85. We do not feel this is a weakness of the calculation for reasons discussed elsewhere 49].

In this paper, we shall discuss only even parity pairing states. This restriction is based on the following experimental evidence for $\mathrm{CeCu}_{2} \mathrm{Si}_{2}$ : the need for strong Pauli limiting to fit the low temperature upper critical field data, $H_{c 2}(0)$ [23]; the reduced spin susceptibility below $T_{c}$ as measured by the ${ }^{63} \mathrm{Cu}$ Knight shift [50], and the observed $T^{3}$ temperature dependence of the nuclear-spin relaxation rate below $T_{c}$ 50]. The strong Pauli limiting actually only argues against equal spin pairing states. As Ueda and Rice showed[51], in the presence of spin-orbit coupling, Pauli limiting is possible for pairing states of $T_{1 u}$ or $T_{2 u}$ symmetry. (Both of these oddparity states would have a gap with point nodes as opposed to line nodes on the Fermi surface.) These experimental facts put together, however, might be considered reasonable evidence for even-parity pairing in $\mathrm{CeCu}_{2} \mathrm{Si}_{2}$.

We have found it useful to study the properties of the scattering amplitude in two steps. First, by setting the functions $I_{s s \Gamma \Gamma^{\prime}}(\vec{q}), I_{s \lambda \Gamma \Gamma^{\prime}}(\vec{q})$, and $I_{\lambda \lambda \Gamma \Gamma^{\prime}}(\vec{q})$ to zero, we simplify the problem considerably to that of two quasiparticles scattering via 
exchange of a momentum independent Boson. In real space, this corresponds to a local interaction between the quasiparticles. We find these local interactions, when averaged over the Fermi surface, are substantially different from those calculated within the jellium model by Zhang and T. K. Lee[17].

In the case of cubic symmetry, inclusion of the functions $I_{s s \Gamma \Gamma^{\prime}}(\vec{q}), I_{s \lambda \Gamma \Gamma^{\prime}}(\vec{q})$, and $I_{\lambda \lambda \Gamma \Gamma^{\prime}}(\vec{q})$ is the computationally intensive part of this calculation. If there are strong local repulsive interactions in the $\eta$ pairing channel, then the only way to get a pairing instability $\left(\right.$ i.e. $\Gamma_{\eta}<0$ ), is to have the functions $I_{s s \Gamma \Gamma^{\prime}}(\vec{q}), I_{s \lambda \Gamma \Gamma^{\prime}}(\vec{q})$, and $I_{\lambda \lambda \Gamma \Gamma^{\prime}}(\vec{q})$, which represent the effect of non-local interactions, overcome the repulsion. Zhang and Lee discovered that in spherical symmetry these q-dependent contributions are too weak to overcome the local repulsions in the $s, d$, and $g$-wave pairing states. We find that in cubic symmetry (with crystal-field splitting) attractive nonlocal interactions can overpower local repulsions in the $T_{1 g}$ pairing channel, thus giving evidence for a $T_{1 g}$ pairing instability.

When we evaluate the diagrams of Figure 13, we can write the quasiparticle scattering amplitude in the even-parity (pseudospin singlet) channel as

$$
\begin{gathered}
\Gamma_{Q P}\left(\vec{k}, \vec{k}^{\prime}\right)=\frac{1}{4} \sum_{\Gamma \alpha \Gamma^{\prime} \alpha^{\prime} \sigma \sigma^{\prime}} \frac{A_{1}^{2}(\vec{k}) A_{1}^{2}\left(\vec{k}^{\prime}\right) \tilde{s}_{o \Gamma} \tilde{s}_{o \Gamma^{\prime}} \tilde{V}_{\Gamma \alpha \sigma^{\prime}}^{*}\left(\vec{k}^{\prime}\right) \tilde{V}_{\sigma^{\prime} \Gamma^{\prime} \alpha^{\prime}}\left(\vec{k}^{\prime}\right) \tilde{V}_{\Gamma^{\prime} \alpha^{\prime} \sigma}^{*}(\vec{k}) \tilde{V}_{\sigma \Gamma \alpha}(\vec{k})}{\left(\epsilon_{\Gamma}-E_{1 \vec{k}}\right)\left(\epsilon_{\Gamma^{\prime}}-E_{1 \vec{k}}\right)} \\
\times\left[D_{s s \Gamma \Gamma^{\prime}}\left(\vec{k}^{\prime}-\vec{k}\right)+D_{s s \Gamma \Gamma^{\prime}}\left(-\vec{k}^{\prime}-\vec{k}\right)-i \frac{\tilde{s}_{o \Gamma}}{\epsilon_{\Gamma}-E_{1 \vec{k}^{\prime}}}\left(D_{s \lambda \Gamma^{\prime}}\left(\vec{k}^{\prime}-\vec{k}\right)+D_{s \lambda \Gamma^{\prime}}\left(-\vec{k}^{\prime}-\vec{k}\right)\right)\right. \\
-i \frac{\tilde{s}_{o \Gamma^{\prime}}}{\epsilon_{\Gamma^{\prime}}-E_{1 \vec{k}^{\prime}}}\left(D_{s \lambda \Gamma}\left(\vec{k}^{\prime}-\vec{k}\right)+D_{s \lambda \Gamma}\left(-\vec{k}^{\prime}-\vec{k}^{\prime}\right)\right) \\
\left.-\frac{\tilde{s}_{o \Gamma} \tilde{s}_{o \Gamma^{\prime}}}{\left(\epsilon_{\Gamma}-E_{1 \vec{k}^{\prime}}\right)\left(\epsilon_{\Gamma^{\prime}}-E_{1 \vec{k}^{\prime}}\right.}\left(D_{\lambda \lambda}\left(\vec{k}^{\prime}-\vec{k}\right)+D_{\lambda \lambda}\left(-\vec{k}^{\prime}-\vec{k}\right)\right)\right]
\end{gathered}
$$

where the symbols mean the following: 
- $\tilde{V}_{\sigma \Gamma \alpha}(\vec{k})=\sqrt{N_{\Gamma}} V_{\sigma \Gamma \alpha}$ is the hybridization matrix element between a plane wave conduction state of momentum $\vec{k}$ and spin $\sigma$ and a crystal field state with quantum numbers $\Gamma$ and $\alpha . N_{\Gamma}$ labels the degeneracy of the $\Gamma$ multiplet.

- $\tilde{s}_{o \Gamma}=s_{o} / \sqrt{N_{\Gamma}}$ can be thought of as the mean field hybridization renormalization coefficient. At mean field level, the bare hybridization is renormalized (due to the constraint of only allowing hopping onto an empty $4 f$ site) to the value $\tilde{s}_{\sigma \Gamma} \tilde{V}_{\sigma \Gamma \alpha}$.

- $\epsilon_{\Gamma}$ is the self-consistent, shifted mean field energy of the $\Gamma$ multiplet.

- $E_{1 \vec{k}}$ is the quasiparticle energy for the lowest, or first, band as a function of momentum.

- $A_{1}^{2}(\vec{k})$ is the quasiparticle normalization function defined in equation 23, and which is very strongly peaked at the six points where the Brillouin zone axes intersect the Fermi surface.

-The components of the Bosonic Green function are:

$$
\begin{aligned}
D_{s s \Gamma \Gamma^{\prime}}(\vec{q}, \tau) & \equiv\left\langle\delta \tilde{s}_{-\vec{q} \Gamma}(\tau) \delta \tilde{s}_{\vec{q} \Gamma^{\prime}}(0)\right\rangle ; \\
D_{s \lambda \Gamma}(\vec{q}, \tau) & \equiv\left\langle\delta \tilde{s}_{-\vec{q} \Gamma}(\tau) \delta \lambda_{\vec{q}}(0)\right\rangle ; \\
D_{\lambda \lambda}(\vec{q}, \tau) & \equiv\left\langle\delta \lambda_{-\vec{q}}(\tau) \delta \lambda_{\vec{q}}(0)\right\rangle .
\end{aligned}
$$

Note that we are taking the static limit of these Green functions. It became clear to us that including the frequency dependence of the boson Green functions was impossible, given the difficult numerical integrals encountered even in the static limit.

When evaluated on the Fermi surface (at zero temperature), the band energies $E_{1 \vec{k}}$ and $E_{1 \vec{k}^{\prime}}$ are set equal to the quasiparticle chemical potential, $\mu$. Thus a term 
like $\epsilon_{\Gamma}-E_{1 \vec{k}}$ becomes

$$
\epsilon_{\Gamma}-\mu=T_{o \Gamma}
$$

which is the Kondo temperature of the $\Gamma$ multiplet. For the case of $\mathrm{CeCu}_{2} \mathrm{Si}_{2}$, the Kondo temperature of the $\Gamma_{7}$ doublet is approximately $10 \mathrm{~K}$, while for the $\Gamma_{8}$ quartet,

$$
T_{o 8}=T_{o 7}+\Delta_{C E F}=370 K
$$

This explains how to treat all the energy denominators in equation 86 .

As mentioned, it is instructive to consider the so-called local limit of equation 86 in which the self-energy functions $I_{s s}(\vec{q}), I_{s \lambda}(\vec{q})$, and $I_{\lambda \lambda}(\vec{q})$ are set to zero,

$$
\begin{aligned}
& \Gamma_{\text {local }}\left(\vec{k}, \vec{k}^{\prime}\right)=\frac{1}{4} \sum_{\Gamma \alpha \Gamma^{\prime} \alpha^{\prime} \sigma \sigma^{\prime}} \frac{A_{1}^{2}(\vec{k}) A_{1}^{2}\left(\vec{k}^{\prime}\right) \tilde{s}_{o \Gamma} \tilde{s}_{o \Gamma^{\prime}} \tilde{V}_{\Gamma \alpha \sigma}^{*}\left(\vec{k}^{\prime}\right) \tilde{V}_{\sigma^{\prime} \Gamma^{\prime} \alpha^{\prime}}\left(\vec{k}^{\prime}\right) \tilde{V}_{\Gamma^{\prime} \alpha^{\prime} \sigma}^{*}(\vec{k}) \tilde{V}_{\sigma \Gamma \alpha}(\vec{k})}{T_{o \Gamma} T_{o \Gamma^{\prime}}} \\
& \times 2\left[\frac{\tilde{s}_{o \Gamma}}{\sqrt{N_{\Gamma^{\prime}}} T_{o \Gamma} \Gamma_{o s \lambda}}+\frac{\tilde{s}_{o \Gamma^{\prime}}}{\sqrt{N_{\Gamma}} T_{o \Gamma^{\prime}} \Gamma_{o s \lambda}}-\frac{\Gamma_{o \lambda \lambda}}{\sqrt{N_{\Gamma} N_{\Gamma^{\prime}}} \Gamma_{o s \lambda}^{2}}\right],
\end{aligned}
$$

where

$$
\begin{gathered}
\Gamma_{o s \lambda}=\sum_{\Gamma}\left(\frac{1}{2} N_{\Gamma} \tilde{s}_{o \Gamma}+\frac{x_{\Gamma}}{\tilde{s}_{o \Gamma}}\right), \\
\Gamma_{o \lambda \lambda}=\sum_{\Gamma} \frac{y_{\Gamma}}{T_{o \Gamma}} .
\end{gathered}
$$

The combination of normalization functions, $A_{1}^{2}(\vec{k}) A_{1}^{2}\left(\vec{k}^{\prime}\right)$, and the product of the four hybridization matrix elements is due to the anisotropic vertices $\gamma_{\operatorname{mix} \Gamma}\left(\vec{k}, \vec{k}^{\prime}\right)$ and $\gamma_{f \Gamma^{\prime}}\left(\vec{k}, \vec{k}^{\prime}\right)$. The contribution from the Bose Green function is that which remains inside the square brackets in equation 89 .

The local scattering amplitude of equation 89 is much simpler to deal with than the full expression of equation 86. Roughly, the functions $I_{s s}(\vec{q}), I_{s \lambda}(\vec{q})$, and $I_{\lambda \lambda}(\vec{q})$ can be thought of as renormalizing the local interactions. In the next section, we present 
our results for the Fermi surface averaged scattering amplitude, $\Gamma_{\eta}$, both with and without the Bosonic self energy.

\section{RESULTS-LOCAL LIMIT}

We now present our results for the Fermi surface averaged (local) quasiparticle interactions, $\Gamma_{\text {local }, \eta}$, where $\eta=A_{1 g}, E_{g}, T_{1 g}$, or $T_{2 g}$. We have not included pairing states in the $A_{2 g}$ representation (See Tables 4 and 5) for two reasons: (1) the lowest order spherical harmonic present in the cubic harmonic $\Phi_{A_{2 g}}$ is $Y_{6 m}$ [52], and the relatively rapid variation throughout the Brillouin zone of the pair wavefunction would correspond to a pairing state with a high kinetic energy and hence should be less accessible than the pairing states labeled by the other representations; $(2)$ the $A_{2 g}$ state would also require a finer mesh for the Fermi surface average than the one we have used and hence would further increase the (already considerable) overall computing time.

Table 6 gives the ratio $\Gamma_{\text {local }, \eta} / T_{o 7}$ for both mean-field parameter sets (a) and (b). Both parameter sets have Kondo temperatures of about $10 \mathrm{~K}$. Also presented in table 6 is the contribution to the Fermi surface average from the so-called "hot-spots". For the hot-spot contribution, the normalization functions $A_{1}^{2}(\vec{k})$ and $A_{1}^{2}\left(\vec{k}^{\prime}\right)$ in equation 89 were approximated with delta functions in k-space that sampled only the six points on the Fermi surface intersected by the Brillouin zone axes. The integrated weight of the delta functions was chosen to equal the area under the peaks, as shown in figure 4. Such a calculation gives us a feeling for the importance of these special points where the normalization function, $A_{1}(\vec{k})$, is rapidly changing. This contribution is 
labeled by $\Gamma_{\text {local,hot }}$ in Table 6 . We see from Table 6 that

- The $A_{1 g}$ pairing channel has (by-far) the largest (repulsive) local interaction, but it is not dominated by what happens at the "hot-spots".

- In the $E_{g}$ channel, the strong anisotropy of the normalization function, gives rise to a weakly attractive local interaction. In fact, the attractive local interaction here is dominated by the contributions from the hot-spots. We note from Table 5 that a pairing state of $E_{g}$ symmetry transforms like $x^{2}-y^{2}$ or $3 z^{2}-r^{2}$, which have maxima along the directions of the axes. The normalization is also strongly peaked along the axes. Thus the $E_{g}$ states are greatly affected by $A_{1}^{2}(\vec{k})$.

- In the $T_{1 g}$ and $T_{2 g}$ channels, the local interaction is weak and repulsive. Neither symmetry channels can "see" what happens at the "hot-spots", because both cubic harmonics, $\Phi_{T 1 g}$ and $\Phi_{T 2 g}$ vanish identically at these points.

In Table 7 we present for comparison, the results for the Fermi-surface averaged scattering amplitude from Zhang and Lee's 17 jellium model calculation, where Legendre polynomials $\left(P_{\eta}\right)$ play the role of the cubic harmonics. States described by $\eta=0$ are s-wave; $\eta=2$ corresponds to d-wave; and $\eta=4$ is g-wave. Zhang and Lee find all non-zero interactions are repulsive and of about the same strength. As we have said, they also found the inclusion of non-local interactions was not sufficient to overcome the local repulsions. In octahedral symmetry, however, the very weak local interactions in the $E_{g}, T_{1 g}$, and $T_{2 g}$ pairing channels make a superconducing instability likely.

\section{RESULTS-INCLUDING NON-LOCAL INTERACTIONS}


This section discusses our results for the Fermi surface average of the full quasiparticle scattering amplitude, $\Gamma_{Q P}\left(\vec{k}, \vec{k}^{\prime}\right)$, as given in equation 86 . The functions $I_{s s \Gamma \Gamma^{\prime}}(\vec{q})$, $I_{s \lambda \Gamma \Gamma^{\prime}}(\vec{q})$, and $I_{\lambda \lambda \Gamma \Gamma^{\prime}}(\vec{q})$ (see equations $68-70$ ), contain the physics of the screened (by density fluctuations of the coupled conduction- $4 f$ electrons) slave Bosons. The momentum dependence represents the contribution of non-local quasiparticles interactions in real space.

From equations 86 and 85 , we see that we must evaluate the self-energy functions for all unique combinations of $\vec{k} \pm \vec{k}^{\prime}$. This we do separately from the actual averaging process. We store the required values for $I_{s s \Gamma \Gamma^{\prime}}(\vec{q}), I_{s \lambda \Gamma \Gamma^{\prime}}(\vec{q})$, and $I_{\lambda \lambda \Gamma \Gamma^{\prime}}(\vec{q})$ in a look-up table.

We discussed the general convergence properties of the functions $I_{s s \Gamma \Gamma^{\prime}}(\vec{q}), I_{s \lambda \Gamma \Gamma^{\prime}}(\vec{q})$ , and $I_{\lambda \lambda \Gamma \Gamma^{\prime}}(\vec{q})$ in Sec. VIII, where we saw that, due to the complicated effective matrix elements in cubic symmetry (see equation 72), they are very slow to converge as a function of the Brillouin zone mesh size. The slow convergence prompted us to try the following line of attack. We have averaged the scattering amplitude (equations 86) over the Fermi surface with a fixed averaging mesh. We vary, however, the mesh for calculating the Bosonic Green function, as characterized by the parameter $n_{m e s h}$, and study the average interactions as a function of $n_{\text {mesh }}$. With this procedure, it became clear that $n_{\text {mesh }} \approx 50$ is a practical limit of the mesh size. Creating the look-up table for the Bosonic self-energies would require well over a week of runtime on a DEC5100 for anything bigger. Thus, it is important to ask if we can make any conclusions about possible pairing instabilities for $n_{\text {mesh }} \leq 50$.

The values of $\Gamma_{\eta} / T_{o 7}$ for $\eta=A_{1 g}$ and $\eta=T_{2 g}$ are plotted as a function of $n_{\text {mesh }}$ 
in Figure 14 for parameter set (a) and in Figure 16 for parameter set (b). Also on the plots, the local value for the average are marked by horizontal lines for each representation (except $A_{1 g}$ ). The results show the following:

- In the $A_{1 g}$ channel, the interactions are clearly repulsive. The non-local interactions, however, are attractive, since the average local repulsion is of the size $\Gamma_{l o c a l, A 1 g} / T_{o 7}=3.54$, and the inclusion of the momentum dependent Bosonic self-energy gives $\Gamma_{A 1 g} / T_{o 7} \approx$ 1.2. Thus the non-local contribution has reduced the local repulsion by about a factor of two.

- In the $T_{2 g}$ channel, but for a glitch at $n_{m e s h}=23$, the interactions are repulsive for parameter set (a), with $\Gamma_{T 2 g} / T_{o 7} \approx 0.20$. For parameter set (b) (Figure 16), however, it is less clear if the average interaction is attractive or repulsive. Taking the results for both parameter sets together, we believe there is no pairing instability of $T_{2 g}$ symmetry.

The values of $\Gamma_{\eta} / T_{o 7}$ for $\eta=E_{g}$ and $\eta=T_{1 g}$ are plotted in Figure 15 for parameter set (a) and in Figure 17 for parameter set (b).

- The results for $T_{1 g}$ show attractive interactions, with an average value $\Gamma_{T 1 g} / T_{o 7}=$ $0.212 \pm 0.025$ for parameter set (a), and $=-0.226 \pm 0.049$ for parameter set (b).

- The interactions in the $E_{g}$ channel are less well behaved, but are attractive for $25 \leq n_{\text {mesh }} \leq 47$ (for parameter set $\left.(\mathrm{a})\right)$. These numbers point to a possible superconducting instability of $E_{g}$ symmetry, but the results have not converged enough for us to be sure. As a rough guide, using the numbers for $25 \leq n_{\text {mesh }} \leq 47$ would give $\langle\Gamma\rangle_{E g} / T_{o 7} \approx-0.163 \pm 0.054$. The values for parameter set (b), however, have a surprisingly large fluctuation at $n_{m e s h}=31$, which makes it very difficult to make any 
conclusion for $n_{\text {mesh }} \leq 35$. It is clear that the $E_{g}$ states are the most sensitive to the fluctuations due to the anisotropic matrix elements in the Bosonic self-energies. From these results, we see that the $T_{1 g}$ pairing state is the most likely candidate for a pairing instability in cubic symmetry.

\section{DISCUSSION}

As we have said, $n_{m e s h} \approx 50$ is a practical limit on the size of the Bosonic self-energy mesh that we could run on a local workstation. The appearance of fluctuations in the averaged scattering amplitude (Figures 14-17), which limit our conclusions about possible pairing instabilities in cubic symmetry, is not shocking. After all, we know that there are fluctuations in the Bosonic self-energies for $n_{m e s h} \approx 80$ (See figures 1113). As we discussed, such variations are due to the anisotropic matrix elements in the principal value integrals over the Brillouin zone. The zone is divided into a large number of tetrahedra $\left(8 \times\left(n_{\text {mesh }}\right)^{3}\right.$ to be exact $)$, and the matrix elements are assumed constant inside a given tetrahedron. If the matrix elements are sharply peaked in some region of the Brillouin zone, then it is easy to see how fluctuations can occur. If the mesh is constructed so that the matrix elements are evaluated very near a peak, then only a small shift in the mesh is required before the matrix elements will be evaluated at a point far down on the sides of the peak. Thus a small change in mesh size could result in a large change in the evaluated matrix elements. A similar problem can arise in finite size lattice problems, where large variations in results can persist up to very large system sizes [53]. The solution for that particular problem is an average over boundary conditions. Our problem, unfortunately, has no such cure, 
and we must live with reasonable conclusions from the data we are able to gather.

To reiterate, we have been able to draw the following conclusions:

- The best candidate for a pairing instability is in a state of $T_{1 g}\left(x y\left(x^{2}+y^{2}\right)\right)$ symmetry, with $\Gamma_{T 1 g} / T_{o 7}=-0.212 \pm 0.025$.

- The $E_{g}$ channel $\left(x^{2}-y^{2}, 3 z^{2}-r^{2}\right)$ also shows weak signs of an instability, with $\Gamma_{E g} / T_{o 7}=-0.163 \pm 0.054$.

- Quasiparticle interactions are strong and repulsize in the $A_{1 g}$ ("s-wave") pairing channel.

- A pairing instability of $T_{2 g}(x y, x z, y z)$ symmetry also appears highly unlikely. Using the classic weak-coupling equation for the superconducting transition temperature within a given representation, $\eta$, we find (in the $T_{1 g}$ channel)

$$
T_{c}\left(\eta=T_{1 g}\right)=1.13 T_{o 7} e^{T_{o 7} / \Gamma_{T 1 g}} \approx 0.09 \mathrm{~K}
$$

which is smaller than the measured $T_{c}$ of $\mathrm{CeCu}_{2} \mathrm{Si}_{2}$. We did not expect, however, such a weak-coupling calculation to give a quantitatively accurate value for $T_{c}$, which leads us to say a few words about strong versus weak coupling results.

In the case of heavy Fermions, the effective Fermi temperature is of the order of the Kondo temperature, which is also the energy scale of importance for the superconducting glue. That is, on physical grounds there is no reason to believe that only a very thin energy shell (thin compared to the Kondo temperature, $T_{07}$ ) about the Fermi surface is of importance for superconductivity. Thus, a strong-coupling calculation, including the energy dependence of the scattering amplitude, should be performed. Given the complexity of the static problem, however, including the dynamics of the slave Bosons in the presence of crystal fields is not feasible. This does 
not necessarily imply that the pairing instabilities found in the static problem have no meaning. We shall discuss, generally, why this is so.

It is conventional wisdom[54], that near the Fermi surface the quasiparticle selfenergy for heavy Fermions is strongly frequency dependent but only weakly dependent on the magnitude of the momentum, $|\vec{k}|$. This can be understood intuitively as follows. The characteristic energy scale for the quasiparticles is the Kondo temperature, $T_{o 7}$, which is about $10 \mathrm{~K}$. The degeneracy temperature for a typical metal is $T_{F} \approx 10,000 \mathrm{~K}$. The characteristic momentum, however, is set by the Fermi wavevector, $k_{F}$, which for $\mathrm{CeCu}_{2} \mathrm{Si}_{2}$ is the size of a typical metal. Thus, broadly speaking, we expect the quasiparticle self-energy, $\Sigma$, to behave (near the Fermi surface) as

$$
\begin{aligned}
\frac{\partial \Sigma}{\partial \omega} & \approx \mathcal{O}\left(\frac{\Sigma}{T_{o 7}}\right), \\
\frac{\partial \Sigma}{\partial \xi_{\vec{k}}} & \approx \mathcal{O}\left(\frac{\Sigma}{D}\right)
\end{aligned}
$$

where $D$ is the bandwidth of the conduction band. Then, since

$$
\frac{\partial \Sigma}{\partial \omega} \gg \frac{\partial \Sigma}{\partial \xi_{\vec{k}}},
$$

it seems reasonable to ignore the momentum dependence of the self-energy. This intuitive result was reinforced by Millis and Lee[11], who found (in the $S U(N)$ model) that the momentum dependence of the imaginary part of the conduction electron selfenergy (at order $1 / N$ ) is very weak (going as $1 / N^{2}$ ), while the frequency dependence goes as the inverse of the Kondo scale.

If one accepts the dominance of the frequency dependence in the quasiparticle selfenergy, then it seems reasonable to assume that including such dependence in a strong 
coupling calculation (ala McMillan [55]) would serve only to reduce the transition temperature, $T_{c}$. We do not believe that the sign of the average scattering amplitude, $\Gamma_{\eta}$, would be affected by such frequency dependence. Thus, our conclusions about which pairing channels, $\eta$, show a superconducting instability should not be changed as the result of a strong coupling calculation.

Please note that we are not saying that the reduction of the transition temperature due to the frequency dependence of the residual quasiparticle interactions would be identical in structure to the case of electron-phonon coupling [55]. We can only say now that we expect $T_{c}$ to be reduced; we can not give an estimate of how large the reduction would be.

The superconducting instabilities themselves appear to be based heavily on the underlying symmetry of the problem, which does not care if one performs a strong or weak coupling calculation. The fact that our estimated (weak-coupling) transition temperature for the $T_{1 g}$ pairing instability is smaller than the measured value for $\mathrm{CeCu}_{2} \mathrm{Si}_{2}$, is not a surprise. The major purpose of this calculation has not been to give a precise numerical recipe for calculating the $T_{c}$ of heavy Fermion systems. We wished to study the importance of local, or "multiplet", physics upon quasiparticle interactions. Thus the a weak coupling calculation should be a reasonable starting point. 


\section{Acknowledgements}

* Present address: Department of Physics, Monmouth College, Monmouth IL.

The author wishes to thank D. L. Cox for invaluable guidance and support over the duration of this project. The author also wishes to thank the following people for

meaningful discussions: J. W. Wilkins, C. Pennington, M. Alouani, M. Steiner, and Q. Si. This work was supported by a grand from the U. S. Department of Energy, Office of Basic Energy Sciences, Division of Materials Research.

\section{References}

[1] F. Steglich, J. Aarts, C. D. Bredl, W. Lieke, D. Meschede, W. Franz, and H. Schäfer, Phys. Rev. Lett. 43, 1892 (1979).

[2] N. Grewe and F. Steglich, in Handbook on the Physics and Chemistry of RareEarths, vol 14, edited by K. A. Gschneider Jr. and L. Eyring, (North-Holland, Amsterdam, 1990).

[3] G. R. Stewart, Rev. Mod. Phys. 56, 755 (1984).

[4] P. A. Lee, T. M. Rice, J. W. Serene, and J. W. Wilkins, Comments on Condens. Matt. Phys. 12, 99 (1986).

[5] B. Welslau and N. Grewe, Ann. Physik 1, 214 (1992).

[6] P. Fulde, J. Keller, and G. Zwicknagl, Solid State Physics 41, 1 (1988). 
[7] D. Rainer, Physica Scripta T23, 106 (1988).

[8] S. E. Barnes, J. Phys. F 6, 1375 (1976).

[9] N. Read and D. M. Newns, J. Phys. C 16, L1055 (1983).

[10] P. Coleman, Phys. Rev. B 29, 3035 (1984).

[11] A. J. Millis and P. A. Lee, Phys. Rev. B 35, 3394 (1986).

[12] A. Auerbach and K. Levin, Phys. Rev. Lett. 57, 877 (1986).

[13] Z. Tešanović and O. T. Valls, Phys. Rev. B 34, 1918 (1986).

[14] P. Coleman, Phys. Rev. B 35, 5072 (1987).

[15] M. Lavagna, A. J. Millis, and P. A. Lee, Phys. Rev. Lett. 58, 266 (1987).

[16] A. Houghton, N. Read, and H. Won, Phys. Rev. B 35, 5123 (1987).

[17] F. C. Zhang and T. K. Lee, Phys. Rev. B 35, 3651 (1987).

[18] B. Coqblin and J. R. Schrieffer, Phys. Rev. 185, 847 (1969).

[19] Z. Zou and P. W. Anderson, Phys. Rev. Lett. 57, 2073 (1986).

[20] N. Read and D. M. Newns, J. Phys. C 16, 3273 (1983).

[21] S. Horn, E. Holland-Moritz, M. Loewenhaupt, F. Steglich, H. Scheuer, A. Benoit, and J. Flouquet, Phys. Rev. B 23, 3171 (1981). 
[22] C. D. Bredl, W. Lieke, R. Schefzyk, M. Lang, U. Rauchschwalbe, F. Steglich, S. Riegel, R. Felten, G. Weber, J. Klaasse, J. Aarts, and F. R. de Boer, J. Magn. Magn. Mat. 47 \& 48, 30 (1985).

[23] F. Steglich, U. Rauchschwalbe, U. Gottwich, H. M. Mayer, G. Sparn, N. Grewe, U. Poppe, and J. J. M. Franse, J. Appl. Phys. 57, 3054 (1985).

[24] U. Rauchschwalbe, Physica 147B, 1 (1987).

[25] K. R. Lea, M. J. M. Leask, and W. P. Wolff, J. Phys. Chem. Solids 23, 1381 (1962)

[26] J. S. Kang, J. W. Allen, O. Gunnarsson, N. E. Christensen, O. K. Andersen, Y. Lassailly, M. B. Maple, and M. S. Torikachvili, Phys. Rev. B 41, 6610 (1990).

[27] R. D. Parks, M. L. den Boer, S. Raaen, J. L. Smith, and G. P. Williams, Phys. Rev. B 30, 1580 (1984).

[28] D. L. Cox, OSU preprint (1990).

[29] D. L. Cox, private communication.

[30] R. M. Martin, Phys. Rev. Lett. 48, 362 (1982).

[31] see. e.g., P. Fulde, Electronic Correlations in Molecules and Solids, (SpringerVerlag, Berlin, 1991), p. 44.

[32] P. W. Anderson, Phys. Rev. B 30, 1549, 4000 (1984).

[33] F. C. Zhang and T. K. Lee, Phys. Rev. B 34, 8114 (1986). 
[34] T. M. Rice and K. Ueda, Phys. Rev. Lett. 55, 995 (1985).

[35] D. L. Cox, Phys. Rev. Lett. 59, 1240 (1987).

[36] F. C. Zhang and T. K. Lee, Phys. Rev. Lett. 58, 2728 (1987).

[37] G. Aeppli and C. M. Varma, Phys. Rev. Lett. 58, 2729 (1987). 808 (1987).

[38] B. Batlogg, J. P. Remeika, A. S. Cooper, and Z. Fisk, J. Appl. Phys. 55, 2001 (1984).

[39] O. Jepsen and O. K. Andersen, Solid State Commun. 9, 1763 (1971).

[40] G. Lehmann, P. Rennert, M. Taut, and H. Wonn, Phys. Status Solidi 37, K27 (1970).

[41] J. Rath and A. J. Freeman, Phys. Rev. B 11, 2109 (1975).

[42] Per-Anker Lingård, Solid State Commun. 16, 481 (1975).

[43] see, e.g., R. L. Jacobs and D. Lipton, in Computational Methods in Band Theory, edited by P. M. Marcus, J. F. Janak, and A. R. Williams, (Plenum Press, New York, 1971).

[44] B. R. Trees, Ph. D. Thesis (The Ohio State University, 1993) unpublished.

[45] D. L. Cox, Ph. D. Thesis (Cornell University, 1985) unpublished.

[46] G. Gilat, J. Comput. Phys. 10, 432 (1972). 
[47] G. Gilat, in Methods in Computational Physics, vol. 15, edited by G. Gilat, B. J. Alder, S. Fernbach, and M. Rotenberg (Academic, New York, 1976).

[48] Matthew Steiner, private communication.

[49] B. R. Trees and D. L. Cox, to be published in Phys. Rev. B, Rapid Communications.

[50] K. Asayama, Y. Kitaoka, and Y. Kohori, J. Magn. Magn. Mat. 76 \& 77, 449 (1988).

[51] K. Ueda and T. M. Rice, Phys. Rev. B 31, 7114 (1985).

[52] S. L. Altmann and A. P. Cracknell, Rev. Mod. Phys. 37, 19 (1965).

[53] K. -H. Luk and D. L. Cox, Phys. Rev B 41, 4456 (1990).

[54] C. M. Varma, Phys. Rev. Lett. 55, 2723 (1985).

[55] W. L. McMillan, Phys. Rev. 167, 331 (1968). 


\section{TABLE CAPTIONS}

Table 1: Functional forms for the angular dependence of $\mu_{\Gamma \alpha \Gamma^{\prime} \alpha^{\prime}}$ for all possible combinations of the crystal field quantum numbers $\Gamma, \alpha, \Gamma^{\prime}, \alpha^{\prime}$. Note, that by timereversal symmetry, $\mu_{\Gamma \alpha \Gamma^{\prime} \alpha^{\prime}}=\mu_{\Gamma^{*} \alpha^{*} \Gamma^{\prime *} \alpha^{\prime *}}$, where the ${ }^{*}$ denotes the time-reversed pair. For example, $\mu_{71,71}=\mu_{7,-1,7,-1}$. Note, also, that $V_{o \Gamma}(|\vec{k}|)$ represents the dependence of the hybridization strength on the radial component of $\vec{k}$. Any combination of quantum numbers not present in the table or not the time-reversed pair of quantum numbers in the table, will vanish upon summing over the pseudo-spin indices.

Table 2: Self-consistent mean field parameter sets, labeled as (a) and (b). At mean field there are three coupled integral equations, which are solved self-consistently. The input parameters are: the bare hybridization strength, $V_{o}$; the lower edge of the conduction electron band in the absence of hybridization, $-D$; the total number of electrons, $n_{\text {total }}=n_{\text {cond }}+n_{f}$, per unit cell; the bare, or unshifted, energy of the $\Gamma_{7}$ $\left(E_{7}\right)$ and the $\Gamma_{8}\left(E_{8}=E_{7}+\Delta_{C E F}\right)$ multiplets; and the fixed crystal field splitting, $\Delta_{C E F}=360 \mathrm{~K}$. The self-consistent parameters which solve the equations are: the hybridization renormalization coefficient, $s_{o}$, where the mean field renormalized hybridization is $s_{o} V_{o}$; the shifted $\Gamma_{7}\left(\epsilon_{7}\right)$ and $\Gamma_{8}\left(\epsilon_{8}=\epsilon_{7}+\Delta_{C E F}\right)$ multiplet energies; the quasiparticle chemical potential, $\mu$; and the Kondo temperature $T_{o 7} \equiv \epsilon_{7}-\mu$ of the $\Gamma_{7}$ doublet. In both parameters sets (a) and (b), the total number of particles was fixed at $n_{\text {total }}=1.5$. And the input parameters were chosen to give approximate Kondo temperatures of $10 \mathrm{~K}$, i.e. $T_{o 7} \approx 10 \mathrm{~K}$. All energies are measured relative the chemical potential in the absence of hybridization. In both parameter sets, $\Delta_{C E F}=360 \mathrm{~K}$, and 
the unshifted $\Gamma_{7}$ energy is $E_{7}=-2.0 \mathrm{eV}$.

Table 3: The parameters $x_{\Gamma}$ and $y_{\Gamma}$ are for both mean field parameter sets described in Table 2.

Table 4: Realizations of the cubic harmonics, $\Phi_{\eta}$, as linear combinations of the spherical harmonics $Y_{l m}$. For each representation, $\eta$, of cubic symmetry, the expansion was cut off after the lowest set of spherical harmonics with $l>0$.

Table 5: Irreducible representations of the octahedral group, $O$. To get the group $O_{h}$, we add inversions to the allowed symmetry operations, the result of which is that all representations pick up a subscript, $g$ (for even parity) or $u$ (for odd parity).

Table 6: The local ("hard-core") quasiparticle scattering amplitude in the presence of crystal electric fields, $\Gamma_{Q P}$, averaged over a spherical Fermi surface. Results for both mean field parameter sets (a) and (b) are given. See table 5.3 for a discussion of the mean field parameters themselves. The first column gives the representations, labeled by $\eta$, of the group $O_{h}$. The second and third columns are the averaged local scattering amplitudes (divided by the $\Gamma_{7}$ Kondo temperature, $T_{o 7}$ ) for the parameter sets (a) and (b), respectively. The fourth column lists (for parameter set (a)) the contribution to the average from the so-called hot-spots, where the Brillouin zone axes intersect the Fermi surface. In column four, in the $E_{g}$ channel, the attractive interaction seems to be due to these hot-spots; but in the $A_{1 g}$ channel the hot-spots do not dominate, since the full Fermi surface average is large and positive. The average in the $E_{g}$ should be most sensitive to the hot-spots, since that is where the $E_{g}$ cubic harmonics have their maximum value. In the $T_{1 g}$ and $T_{2 g}$ channels there is rigorously zero contribution from the hot-spots because the $T_{1 g}$ and $T_{2 g}$ cubic harmonics vanish 
at those points.

Table 7: The local ("hard-core") quasiparticle scattering amplitude in the jellium model, as studied by Zhang and Lee[1]. When divided by the Kondo temperature and averaged over the spherical Fermi surface, these results are universal; there are no other parameters involved. In spherical symmetry, the pairing states are labeled by their relative angular momentum, $l$, with $l=0$ corresponding the s-wave; $l=2$, dwave; and $l=4$, g-wave. It is meaningful to compare Zhang and Lee's results with ours, because in the limit of spherical symmetry, where all the Bosonic propagators in equation 5.37 are replaced with their values in spherical symmetry, and where the normalization functions, $A_{1}^{2}(\vec{k})$ and $A_{1}^{2}\left(\vec{k}^{\prime}\right)$, are replaced by their isotropic values in spherical symmetry, our expression for $\Gamma_{Q P}$ (equation 5.73) gives exactly the same local interactions as Zhang and Lee. Note that all local interactions are repulsive in spherical symmetry. 


\section{FIGURE CAPTIONS}

Figure 1: Splitting of $J=5 / 2$ multiplet into a $\Gamma_{7}$ doublet and a $\Gamma_{8}$ quartet due to crystal fields of cubic symmetry. Neutron scattering gives a splitting of about 360 K.

Figure 2: A schematic representation of the hybridization process, in which a conduction electron in the state $\mid \vec{k} \sigma>$ jumps into an empty $4 f$ orbital of total angular momentum $J=5 / 2$, and z-component $m$ described by $\mid \mathrm{m}>$. The matrix element is $<\mathrm{m}|\hat{V}| \vec{k} \sigma>=\mathrm{V}_{m \sigma}(\vec{k})$. If we expand the conduction state in partial waves, then only the state with total angular momentum $J=5 / 2$ and z-component $m$ would be able to hybridize. Note that the energy of the $J=7 / 2$ excited multiplet is physically too large; it is shown thus merely to make the figure more readable. The actual size of the spin orbit coupling in $\mathrm{CeCu}_{2} \mathrm{Si}_{2}$, should be about an order of magnitude larger than the crystal field splitting. Note, also, that the energy splittings of the $\Gamma_{6}, \Gamma_{7}$, and $\Gamma_{8}$ states, which come from the crystal field splitting of the $J=7 / 2$ multiplet, are not accurate. The states are shown merely to inform the reader how the $J=7 / 2$ multiplet decomposes in cubic symmetry. See, for example, Appendix D in reference [40].

Figure 3: Schematic of the quasiparticle bandstructure, showing the shifted crystal field multiplet energies $\left(\epsilon_{7}\right.$ and $\left.\epsilon_{8}\right)$, the chemical potential $(\mu)$, and the lower band edge (-D). (a) the $\mathrm{k}$ values range from the zone center (the $\Gamma$ point) to the intersection of the $k_{x}$ axis with the cubic Brillouin zone boundary. (b) From the zone center to the intersection of a cube diagonal with the Brillouin zone boundary. 
Figure 4: A plot of the normalization function $A_{1}^{2}(\vec{k})$ along the equator of a sphere in k-space as a function of azimuthal angle, $\phi$. The sharp spikes occur at the intersections of the equator with the coordinate axes. At these points the $\Gamma_{7}$ hybridization matrix elements vanish exactly, and the width of these peaks is set by the ratio of the two Kondo temperatures $T_{o 7} / T_{o 8} \approx T_{o 7} / \Delta_{C E F}$. The behavior for a fixed azimuthal angle $(\phi=0)$ as a function of the polar angle, $\theta$, is the same.

Figure 5: Plot of the lowest energy band, $E_{1 \vec{k}}$, for the two mean-field parameter sets (labeled as (a) and (b)). In set (a), the conduction electron chemical potential is $2.44 \mathrm{eV}$, and in set (b), it is $3.33 \mathrm{eV}$. Note that the bands are just shifted with respect to each other. For both parameter sets the unshifted $\Gamma_{7}$ multiplet energy sits at $-2.0 \mathrm{eV}$.

Figure 6: A diagrammatic representation of the infinite-order summation that gives the mean-field dressed f-electron propagator. All the Green functions are defined pictorially at the top of the figure. The bare conduction electron propagator is represented by a solid line.

Figure 7: Diagrammatic representation of Dyson's equation for the conduction Green function. The double line is the hybridization dressed conduction Green function, and $\Sigma_{c \sigma}(\vec{k})$ is the conduction self-energy. All other elements of the figure are as defined in Figure 6.

Figure 8: Diagrammatic representation of Dyson's equation for the mixing Green function.

Figure 9: (a). Dyson's equation for the diagonal component (in the crystal field indices) of the inverse of the (matrix) Bosonic propagator $\hat{D}_{\Gamma \Gamma}^{-1}$. (b). The three 
hybridization-dressed (mean-field) Fermionic Green functions. Note that, in general, the $f$ Green function can mix the multiplet indices at k-points away from the zone center. This is because away from the zone center, the symmetry is lower than cubic, allowing the crystal field indices to mix. (c). Leading contributions to the components $(s s, s \lambda$, and $\lambda \lambda)$ of the self-energy matrix from closed Fermionic loops. The " $\times$ " symbol represents the scaled hybridization matrix element, $\tilde{V}_{\Gamma \alpha \sigma}(\vec{k})$, which

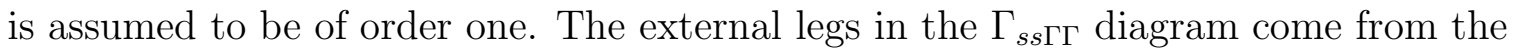
scaled fluctuations in the $s$ fields, $\delta \tilde{s}_{\vec{q} \Gamma}$.

Figure 10: Numerically evaluated function $I_{s s}(\vec{q})$ as a function of the mesh parameter, $n_{\text {mesh }}$ for mean-field parameter set (a). Here we used $\vec{q}=0.5 \hat{z}$.

Figure 11: Numerically evaluated function $I_{s \lambda}(\vec{q})$ as a function of the mesh parameter, $n_{\text {mesh }}$ for mean-field parameter set (a). Here we used $\vec{q}=0.5 \hat{z}$.

Figure 12: Numerically evaluated function $I_{\lambda \lambda}(\vec{q})$ as a function of the mesh parameter, $n_{\text {mesh }}$ for mean-field parameter set (a). Here we used $\vec{q}=0.5 \hat{z}$.

Figure 13: Quasiparticle scattering amplitude for incoming particles (solid lines) of momenta $\vec{k}$ and $-\vec{k}$. The wavy lines are dressed boson propagators, and the vertices denote the anisotropic coupling of quasiparticles to bosons.

Figure 14: Fermi surface averages in the $A_{1 g}$ and $T_{2 g}$ pairing channels of the scattering amplitude, $\langle\Gamma\rangle / T_{o 7}$, as a function of the Boson mesh parameter $n_{m e s h}$. $8 n_{\text {mesh }}^{3}$ equals the number of tetrahedra used in the Brillouin zone integrals for the Bosonic Green functions. These data are for mean field parameter set (a). (See Table 5.3.)

- The horizontal line marked by $T_{2 g, l o c a l}$ denotes the size of the local ("hard-core") 
contribution to the average in the $T_{2 g}$ channel. The corresponding local contribution for the $A_{1 g}$ channel is 3.54 and would be just above the top of the graph. We see that the nonlocal contribution in the $T_{2 g}$ channel is of the same size as the local contribution. Even with the fluctuation at $n_{\text {mesh }}=23$, it seems unlikely there is a pairing instability of $T_{2 g}$ symmetry.

-In the $A_{1 g}$ channel, even with the large fluctuation for $n_{m e s h}=39$, there is clearly no pairing instability.

Figure 15: Fermi surface averages in the $E_{1 g}$ and $T_{1 g}$ pairing channels of the scattering amplitude, $\langle\Gamma\rangle / T_{o 7}$, as a function of the Boson mesh parameter $n_{\text {mesh }}$. These data are for mean field parameter set (a). The solid (dashed) horizontal line denotes the local contribution to the average in the $E_{g}\left(T_{1 g}\right)$ channel.

-Although not yet converged, the averages in the $T_{1 g}$ channel point to the possibility of a pairing instability. Averaging the values of $\langle\Gamma\rangle_{T 1 g} / T_{o 7}$ for all the values of $n_{m e s h}$ used here yields

$$
\frac{\langle\Gamma\rangle_{T 1 g}}{T_{o 7}}=-0.212 \pm 0.025 .
$$

-In the $E_{g}$ channel, there is a large fluctuation at $n_{\text {mesh }}=39$. Therefore, we are hesitant to say that this is evidence of a superconducting instability. However, it is clear that the average interactions in this channel are attractive for a relatively wide range of mesh sizes: $25 \leq n_{\text {mesh }} \leq 47$.

Figure 16: Fermi surface averages in the $A_{1 g}$ and $T_{2 g}$ pairing channels of the scattering amplitude, $\langle\Gamma\rangle / T_{o 7}$, as a function of the Boson mesh parameter $n_{m e s h}$. $\left(8 n_{\text {mesh }}^{3}\right.$ equals the number of tetrahedra used in the Brillouin zone integrals for the 
Bosonic Green functions.) These data are for mean field parameter set (b).

-In the $T_{2 g}$ channel, the horizontal, dashed line denotes the local contribution to the average. The fluctuations in the full average are at least a factor of two larger than the local part and are also varying about zero. Thus it is not possible to say, from the present data, if there is a $T_{2 g}$ instability or not. Using the results from parameter set (a), however, it still seems unlikely that there is an instability in this channel.

-In the $A_{1 g}$ channel, it is easy to see that there average interactions are repulsive and strong. There is no instability in this channel.

Figure 17: Fermi surface averages in the $E_{1 g}$ and $T_{1 g}$ pairing channels of the scattering amplitude, $\langle\Gamma\rangle / T_{o 7}$, as a function of the Boson mesh parameter $n_{m e s h}$. These data are for mean field parameter set (b).

-In the $E_{g}$ channel, the horizontal line denotes the contribution to the average in the local limit. For the full average, up to $n_{m e s h}=31$, the average value is fluctuating evenly about the local value. The surprisingly large fluctuation at $n_{m e s h}=31$, however, makes it impossible to tell if there is an instability in this channel.

-In the $T_{1 g}$ channel, the full average, although fluctuating, remains negative for $21 \leq$ $n_{\text {mesh }} \leq 41$. Averaging these values gives a result of

$$
\frac{\langle\Gamma\rangle_{T 1 g}}{T_{o 7}}=-0.226 \pm 0.049
$$




\begin{tabular}{|c|c|c|}
\hline$\Gamma, \alpha$ & $\Gamma^{\prime}, \alpha^{\prime}$ & $\mu_{\Gamma \alpha \Gamma^{\prime} \alpha^{\prime}}(\vec{k}) / \tilde{V}_{o \Gamma}(|\vec{k}|) \tilde{V}_{o \Gamma^{\prime}}(|\vec{k}|)$ \\
\hline 7,1 & 7,1 & $-\frac{2 \sqrt{\pi}}{3}\left[Y_{00}-\frac{1}{3} Y_{40}-\frac{1}{3} \sqrt{\frac{5}{14}}\left(Y_{44}+Y_{4-4}\right)\right]$ \\
\hline 8,2 & 8,2 & $-\frac{2 \sqrt{\pi}}{3}\left[Y_{00}-\frac{8}{7} \sqrt{\frac{1}{5}} Y_{20}+\frac{1}{21} Y_{40}+\frac{1}{3} \sqrt{\frac{5}{14}}\left(Y_{44}+Y_{4-4}\right)\right]$ \\
\hline 8,1 & 8,1 & $-\frac{2 \sqrt{\pi}}{3}\left[Y_{00}+\frac{8}{7} \sqrt{\frac{1}{5}} Y_{20}+\frac{2}{7} Y_{40}\right]$ \\
\hline 8,2 & 8,1 & $\frac{10}{21} \sqrt{\frac{2 \pi}{5}}\left[Y_{22}+\frac{3}{5} Y_{2-2}-\frac{\sqrt{3}}{2} Y_{42}+\frac{\sqrt{3}}{6} Y_{4-2}\right]$ \\
\hline 8,1 & 8,2 & $\frac{10}{21} \sqrt{\frac{2 \pi}{5}}\left[Y_{2-2}+\frac{3}{5} Y_{22}-\frac{\sqrt{3}}{2} Y_{4-2}+\frac{\sqrt{3}}{6} Y_{42}\right]$ \\
\hline 8,2 & $8,-1$ & $-\frac{4}{21} \sqrt{\frac{2 \pi}{5}}\left[Y_{2-1}+\frac{5}{6} \sqrt{\frac{3}{2}} Y_{4-1}-\frac{5}{6} \sqrt{\frac{21}{2}} Y_{43}\right]$ \\
\hline $8,-1$ & 8,2 & $\frac{4}{21} \sqrt{\frac{2 \pi}{5}}\left[Y_{21}+\frac{5}{6} \sqrt{\frac{3}{2}} Y_{4-1}-\frac{5}{6} \sqrt{\frac{21}{2}} Y_{4-3}\right]$ \\
\hline 7,1 & 8,2 & $\frac{4}{21} \sqrt{6 \pi}\left[Y_{2-1}-\sqrt{\frac{1}{6}} Y_{4-1}\right]$ \\
\hline 8,2 & 7,1 & $-\frac{4}{21} \sqrt{6 \pi}\left[Y_{21}-\sqrt{\frac{1}{6}} Y_{41}\right]$ \\
\hline 7,1 & 8,1 & $-\frac{4}{21} \sqrt{2 \pi}\left[Y_{21}+\frac{1}{6} \sqrt{\frac{21}{2}} Y_{4-3}+\frac{5}{6} \sqrt{\frac{3}{2}} Y_{41}\right]$ \\
\hline 8,1 & 7,1 & $-\frac{4}{21} \sqrt{2 \pi}\left[Y_{2-1}+\frac{1}{6} \sqrt{21} 2 Y_{43}+\frac{5}{6} \sqrt{\frac{3}{2}} Y_{4-1}\right]$ \\
\hline 7,1 & $8,-1$ & $\frac{2}{21} \sqrt{18 \pi}\left[Y_{22}-\frac{1}{3} Y_{2-2}+\frac{1}{2 \sqrt{3}} Y_{4-2}+\frac{5}{6 \sqrt{3}} Y_{42}\right]$ \\
\hline $8,-1$ & 7,1 & $\frac{2}{21} \sqrt{18 \pi}\left[Y_{2-2}-\frac{1}{3} Y_{22}+\frac{1}{2 \sqrt{3}} Y_{42}+\frac{5}{6 \sqrt{3}} Y_{4-2}\right]$ \\
\hline 7,1 & $8,-2$ & $-\frac{4}{21} \sqrt{\pi}\left[Y_{20}-\frac{\sqrt{5}}{3} Y_{40}+\frac{5}{6} \sqrt{\frac{7}{2}} Y_{4-4}-\frac{1}{6} \sqrt{\frac{7}{2}} Y_{44}\right]$ \\
\hline $8,-2$ & 7,1 & $-\frac{4}{21} \sqrt{\pi}\left[Y_{20}-\frac{\sqrt{5}}{3} Y_{40}+\frac{5}{6} \sqrt{\frac{7}{2}} Y_{44}-\frac{1}{6} \sqrt{\frac{7}{2}} Y_{4-4}\right]$ \\
\hline
\end{tabular}




\begin{tabular}{|c|c|c|c|c|c|c|}
\hline \hline $\begin{array}{c}\text { parameter } \\
\text { set }\end{array}$ & $\begin{array}{c}V_{o} \\
(\mathrm{eV})\end{array}$ & $s_{o}$ & $\begin{array}{c}\epsilon_{7} \\
(\mathrm{eV})\end{array}$ & $\begin{array}{c}\mu \\
(\mathrm{eV})\end{array}$ & $\begin{array}{c}T_{o 7} \\
(\mathrm{~K})\end{array}$ & $\begin{array}{c}D \\
(\mathrm{eV})\end{array}$ \\
\hline $\mathrm{a}$ & 0.8595 & 0.093472 & 0.0217648 & 0.0208399 & 9.25 & 2.4405 \\
\hline $\mathrm{b}$ & 0.650 & 0.142409 & -0.8437021 & -0.844999 & 13.0 & 3.33856 \\
\hline \hline
\end{tabular}




\begin{tabular}{|c|c|c|c|c|}
\hline \hline parameter set & $x_{7}$ & $x_{8}$ & $y_{7}$ & $y_{8}$ \\
\hline $\mathrm{a}$ & 1.820 & 0.0717 & 0.975 & 0.04029 \\
\hline $\mathrm{b}$ & 1.729 & 0.0949 & 0.925 & 0.0498 \\
\hline \hline
\end{tabular}




\begin{tabular}{|c|c|}
\hline$\eta$ & $\Phi_{\eta}$ \\
\hline$A_{1 g}$ & $\frac{1}{\sqrt{2}}\left(Y_{00}+0.76376261 Y_{40}+0.4564355\left(Y_{44}+Y_{4-4}\right)\right)$ \\
\hline$A_{2 g}$ & $0.58630197\left(Y_{62}+Y_{6-2}\right)-0.3952847\left(Y_{66}+Y_{6-6}\right)$ \\
\hline$E_{g}$ & $Y_{20}$ \\
$E_{g}$ & $\frac{1}{\sqrt{2}}\left(Y_{22}+Y_{2-2}\right)$ \\
\hline$T_{1 g}$ & $\left(\frac{-0.935414355}{\sqrt{2}}\left(Y_{41}-Y_{4-1}\right)-\frac{0.3535533912}{\sqrt{2}}\left(Y_{43}-Y_{4-3}\right)\right)$ \\
$T_{1 g}$ & $\left(\frac{0.93541435}{\sqrt{2}}\left(Y_{41}+Y_{4-1}\right)-\frac{-0.353553391}{\sqrt{2}}\left(Y_{43}+Y_{4-3}\right)\right)$ \\
$T_{1 g}$ & $\frac{i}{\sqrt{2}}\left(Y_{44}-Y_{4-4}\right)$ \\
\hline$T_{2 g}$ & $\frac{i}{\sqrt{2}}\left(Y_{21}-Y_{2-1}\right)$ \\
$T_{2 g}$ & $\frac{1}{\sqrt{2}}\left(Y_{21}+Y_{2-1}\right)$ \\
$T_{2 g}$ & $\frac{i}{\sqrt{2}}\left(Y_{22}-Y_{2-2}\right)$ \\
\hline
\end{tabular}


$\underline{\text { Representation Dimensionality }} \quad \underline{\text { Transforms like }}$

$\begin{array}{ccc}A_{1} & 1 & \frac{T y^{2}+y^{2}+z^{2}}{\left(x^{2}-y^{2}\right)\left(z^{2}-x^{2}\right)\left(y^{2}-z^{2}\right)} \\ A_{2} & 1 & x^{2}-y^{2}, 3 z^{2}-r^{2} \\ E & 2 & x, y, z \\ T_{1} & 3 & x y, y z, z x \\ T_{2} & 3 & \end{array}$




\begin{tabular}{|c|c|c|c|}
\hline (a) Cubic Symmetry & $\left\langle\Gamma_{\text {local }}\right\rangle_{\eta} / T_{o 7}$ & $\left\langle\Gamma_{\text {local }}\right\rangle_{\eta} / T_{o 7}$ & $\left\langle\Gamma_{\text {local }, \text { hot }}\right\rangle / T_{o 7}$ \\
$\eta$ & set (a) & set (b) & set (a) \\
\hline$A_{1 g}(\mathrm{~s}-$ wave $)$ & 3.54 & 3.53 & -0.215 \\
$E_{g}\left(d_{x^{2}-y^{2}}, d_{3 z^{2}-r^{2}}\right)$ & -0.0275 & -0.0453 & -0.0696 \\
$T_{1 g}$ & 0.0478 & 0.0695 & 0.0 \\
$T_{2 g}\left(d_{x y}, d_{y z}, d_{x z}\right)$ & 0.0487 & 0.0709 & 0.0 \\
\hline
\end{tabular}




\begin{tabular}{|c|c|}
\hline Spherical Symmetry & $\left\langle\Gamma_{\text {local }}\right\rangle_{\eta} / T_{o}$ \\
\hline$\eta=0$ (s-wave) & $1 / 3$ \\
$\eta=2$ (d-wave) & $8 / 21$ \\
$\eta=4$ (g-wave) & $2 / 7$ \\
\hline
\end{tabular}

